\title{
SOUS-GROUPE DE BRAUER INVARIANT ET OBSTRUCTION DE DESCENTE ITÉRÉE
}

\author{
YANG CAO
}

\begin{abstract}
RÉSumÉ. Pour une variété quasi-projective, lisse, géométriquement intègre sur un corps de nombres $k$, on montre que l'obstruction de descente itérée est équivalente à l'obstruction de descente. Ceci généralise un résultat de Skorobogatov, et ceci répond à une question ouverte de Poonen. Les outils principaux sont la notion de sous-groupe de Brauer invariant et la notion d'obstruction de Brauer-Manin étale invariante pour une $k$-variété munie d'une action d'un groupe linéaire connexe.

Summary. For a quasi-projective smooth geometrically integral variety over a number field $k$, we prove that the iterated descent obstruction is equivalent to the descent obstruction. This generalizes a result of Skorobogatov, and this answers an open question of Poonen. Our main tools are the notion of invariant Brauer subgroup and the notion of invariant étale Brauer-Manin obstruction for a $k$-variety equipped with an action of a connected linear algebraic group.
\end{abstract}

\section{TABLE DES MATIÈRES}

1. Introduction 1

2. Torseur universel de n-torsion et formule de Künneth de degré 2

3. Préliminaires sur les torseurs sous un groupe fini 11

3.1. Torseur sur un groupe algébrique 11

3.2. Relèvement d'une action par un torseur 12

3.3. Le groupe minimal compatible avec un torseur 15

4. Rappel sur le sous-groupe de Brauer invariant 16

5. La descente par rapport au sous-groupe de Brauer invariant 18

6. Démonstration du théorème 1.4 24

7. Démonstration des théorèmes 1.1 et $1.2 \quad 28$

Références 28

\section{INTRODUCTION}

Soit $k$ un corps de nombres. Soit $\mathbf{A}_{k}$ l'anneau des adèles de $k$. Pour une $k$-variété lisse $X$, on note $X\left(\mathbf{A}_{k}\right)$ l'ensemble des points adéliques de $X$. On a le plongement diagonal

$$
X(k) \subset X\left(\mathbf{A}_{k}\right) .
$$

C'est une question importante de caractériser l'adhérence des points rationnels dans les points adéliques (principe de Hasse, approximation faible, approximation forte). Manin en 1970 a

Date: 20 mars 2020. 
montré que cette adhérence est contenue dans un fermé déterminé par le groupe de Brauer de la variété $X$ ([Ma]). Depuis lors, divers auteurs (Manin, Colliot-Thélène, Sansuc, Skorobogatov, Harari, Demarche) ont décrit d'autres fermés de $X\left(\mathbf{A}_{k}\right)$ contenant les points rationnels, et se sont attachés à comprendre les inclusions entre ces divers fermés. On a utilisé pour cela les torseurs sous des groupes linéaires (finis ou non) sur $X$, et on a utilisé des combinaisons de ces deux approches pour déterminer des fermés minimaux de $X\left(\mathbf{A}_{k}\right)$ contenant $X(k)$. Harari et Skorobogatov ont décrit une inclusion ([HS02, Déf. 4.2], cf. (1.2) pour la définition)

$$
X(k) \subset X\left(\mathbf{A}_{k}\right)^{\text {descent }} .
$$

Ensuite Poonen a itéré cette inclusion en ([Po, §8.5.2], cf. (1.3) pour la définition)

$$
X(k) \subset X\left(\mathbf{A}_{k}\right)^{\text {descent,descent }} \subset X\left(\mathbf{A}_{k}\right)^{\text {descent }},
$$

et demandé (cf. [Po, §8.5.4]) si la deuxième inclusion raffine la première. Le théorème principal du présent article (théorème 1.1) permet de répondre à cette question de Poonen : $X\left(\mathbf{A}_{k}\right)^{\text {descent,descent }}=X\left(\mathbf{A}_{k}\right)^{\text {descent }}$ (théorème 1.2 ci-dessous). Ce théorème 1.2 apporte un point final à l'utilisation combinée du groupe de Brauer et de la descente sous des groupes linéaires dans la détermination de l'adhérence de $X(k)$ dans $X\left(\mathbf{A}_{k}\right)$.

Donnons maintenant des énoncés précis.

On note $\Omega_{k}$ l'ensemble des places du corps de nombres $k$. Pour chaque $v \in \Omega_{k}$, on note $k_{v}$ le complété de $k$ en $v$ et $\mathcal{O}_{v} \subset k_{v}$ l'anneau des entiers $\left(\mathcal{O}_{v}=k_{v}\right.$ pour $v$ archimédienne).

Pour $B$ un sous-groupe de $\operatorname{Br}(X)$, on définit

$$
X\left(\mathbf{A}_{k}\right)^{B}=\left\{\left(x_{v}\right)_{v \in \Omega_{k}} \in X\left(\mathbf{A}_{k}\right): \sum_{v \in \Omega_{k}} i n v_{v}\left(\xi\left(x_{v}\right)\right)=0 \in \mathbb{Q} / \mathbb{Z}, \quad \forall \xi \in B\right\} .
$$

Comme l'a remarqué Manin ([Ma]), la théorie du corps de classes donne $X(k) \subseteq X\left(\mathbf{A}_{k}\right)^{B}$.

Soient $F$ un $k$-groupe algébrique et $f: Y \rightarrow X$ un $F$-torseur. Pour tout 1-cocycle $\sigma \in$ $Z^{1}(k, F)$, on note $F_{\sigma}$, respectivement $f_{\sigma}: Y_{\sigma} \rightarrow X$ le tordu du $k$-groupe $F$, respectivement du torseur $f$, par le 1-cocycle $\sigma$. Alors $f_{\sigma}$ est un $F_{\sigma}$-torseur. La classe d'isomorphisme du $k$-groupe $F_{\sigma}$, respectivement du torseur $f_{\sigma}$, ne dépend que de la classe de $\sigma$ dans $H^{1}(k, F)$. Par abus de notation, étant donnée une classe $[\sigma] \in H^{1}(k, F)$, on note $F_{\sigma}=F_{[\sigma]}$ et $f_{\sigma}=f_{[\sigma]}$.

Pour une $k$-variété lisse $X$, Skorobogatov ([Sk99]) et Poonen définissent ([Po10, §3.3]) l'ensemble suivant

$$
X\left(\mathbf{A}_{k}\right)^{\mathrm{e} t, \mathrm{Br}}:=\bigcap_{\substack{f: Y \\ F \text { fini }}} \bigcup_{\vec{F}_{X},} \bigcup_{\sigma \in H^{1}(k, F)} f_{\sigma}\left(Y_{\sigma}\left(\mathbf{A}_{k}\right)^{\operatorname{Br}\left(Y_{\sigma}\right)}\right),
$$

où $F$ parcourt les $k$-groupes finis. Ils obtiennent une inclusion $X(k) \subset X\left(\mathbf{A}_{k}\right)^{\text {ét,Br }}$. Ceci définit une obstruction au principe de Hasse pour $X$, appelée obstruction de Brauer-Manin étale, étudiée dans le cas projectif par Skorobogatov, Harari et Demarche, puis dans le cas quasiprojectif [CDX].

Le résultat principal de cet article est :

Théorème 1.1. Soient $G$ un k-groupe linéaire quelconque, $Z$ une $k$-variété lisse et $p: X \rightarrow Z$ un G-torseur. Alors :

$$
Z\left(\mathbf{A}_{k}\right)^{\text {ét,Br }}=\cup_{\sigma \in H^{1}(k, G)} p_{\sigma}\left(X_{\sigma}\left(\mathbf{A}_{k}\right)^{\text {ét,Br }}\right) .
$$


Pour $G$ fini et $Z$ projective, ce théorème avait déjà été établi par Skorobogatov ([Sk09, Thm. 1.1]). Pour $G$ fini et $Z$ quasi-projective, il avait été ensuite établi par Demarche, Xu et l'auteur ([CDX, Prop. 6.6]). Si $Z$ est projective, $\pi_{1}\left(Z_{\bar{k}}\right)$ est fini et $G$ est une extension d'un $k$-groupe fini par un tore, Balestrieri a établi une variante simple dans [Ba, Thm. 1.9], où elle considère l'obstruction de Brauer-Manin algébrique étale.

Par ailleurs, dans $[\mathrm{Po} 10, \S 3.2]$ et $[\mathrm{Po}, \S 8]$, on définit deux ensembles

$$
\begin{aligned}
& X\left(\mathbf{A}_{k}\right)^{\text {descent }}:=\bigcap_{\substack{f: Y \stackrel{F}{\rightarrow} X_{X}, F \text { linéaire }}} \bigcup_{\sigma \in H^{1}(k, F)} f_{\sigma}\left(Y_{\sigma}\left(\mathbf{A}_{k}\right)\right),
\end{aligned}
$$

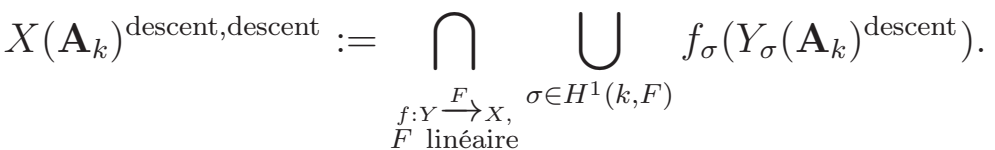

On a $X(k) \subset X\left(\mathbf{A}_{k}\right)^{\text {descent }}$ et $X(k) \subset X\left(\mathbf{A}_{k}\right)^{\text {descent,descent }}$. Ceci définit deux nouvelles obstructions au principe de Hasse pour $X$, appelées obstruction de descente et obstruction de descente itérée. D'après la série de travaux ([D09a], [Sk09] et $[\mathrm{CDX}])$, on a $X\left(\mathbf{A}_{k}\right)^{\text {ét,Br }}=X\left(\mathbf{A}_{k}\right)^{\text {descent }}$ lorsque $X$ est quasi-projective ([CDX, Thm. 1.5]). Du théorème 1.1 on déduit facilement le :

Théorème 1.2. Pour toute variété quasi-projective lisse géométriquement intègre $X$, on a $X\left(\mathbf{A}_{k}\right)^{\text {descent, descent }}=X\left(\mathbf{A}_{k}\right)^{\text {descent }}$.

L'idée clé de la démonstration du théorème 1.1 est la notion de sous-groupe de Brauer invariant ([C18, Déf. 3.1]), que nous rappelons ici :

Définition 1.3. Soit $G$ un groupe algébrique connexe.

(1) Soit $(X, \rho)$ une $G$-variété lisse connexe. Le sous-groupe de Brauer $G$-invariant de $X$ est le sous-groupe

$$
\operatorname{Br}_{G}(X):=\left\{b \in \operatorname{Br}(X):\left(\rho^{*}(b)-p_{2}^{*}(b)\right) \in p_{1}^{*} \operatorname{Br}(G)\right\}
$$

de $\operatorname{Br}(X)$, où $G \times X \stackrel{p_{1}}{\rightarrow} G, G \times X \stackrel{p_{2}}{\rightarrow} X$ sont les projections et $G \times X \stackrel{\rho}{\rightarrow} X$ est l'action de $G$.

(2) Soit $X$ une $G$-variété lisse quelconque. Le sous-groupe de Brauer $G$-invariant de $X$ est le sous-groupe $\operatorname{Br}_{G}(X) \subset \operatorname{Br}(X)$ des éléments $\alpha$ vérifiant $\left.\alpha\right|_{X^{\prime}} \in \operatorname{Br}_{G}\left(X^{\prime}\right)$ pour toute composante connexe $X^{\prime}$ de $X$.

(3) Soient $F$ un $k$-groupe fini et $X$ une $G$-variété lisse quelconque. Un $F$-torseur $Y \stackrel{f}{\rightarrow} X$ est $G$-compatible s'il existe une action de $G$ sur $Y$ telle que $f$ soit un $G$-morphisme.

D'après la proposition 3.3, l'action de $G$ sur $Y$ vérifiant les conditions ci-dessus est unique et le $F_{\sigma}$-torseur $f_{\sigma}$ est aussi $G$-compatible pour tout $\sigma \in H^{1}(k, F)$. On définit la variante de $X\left(\mathbf{A}_{k}\right)^{\text {ét,Br }}$ suivante :

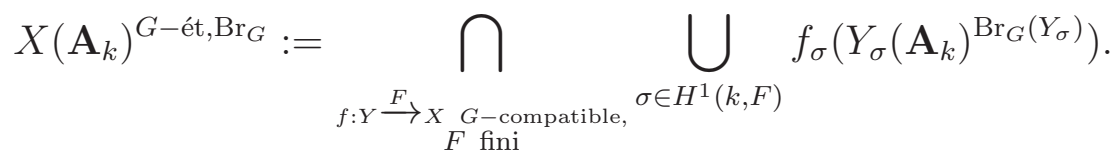

Alors $X(k) \subset X\left(\mathbf{A}_{k}\right)^{\text {ét,Br }} \subset X\left(\mathbf{A}_{k}\right)^{G \text {-ét,Br}}{ }_{G}$. Ceci définit une obstruction au principe de Hasse pour $X$, appelée obstruction de Brauer-Manin étale invariante.

Le théorème suivant joue un rôle clé dans la démonstration du théorème 1.1. 
Théorème 1.4. Soient $G$ un groupe linéaire connexe et $X$ une $G$-variété lisse. Alors

$$
X\left(\mathbf{A}_{k}\right)^{G-e ́ t, B r}{ }_{G}=X\left(\mathbf{A}_{k}\right)^{\text {ét,Br }} .
$$

Dans le cas où $X$ est un $G$-espace homogène à stabilisateur géométrique connexe, d'après le corollaire 3.5 (4), tout torseur $G$-compatible sous un $k$-groupe fini est constant. Donc on peut obtenir facilement le résultat suivant.

Corollaire 1.5. Soient $G$ un groupe linéaire connexe et $X$ un $G$-espace homogène à stabilisateur géométrique connexe. Alors

$$
X\left(\mathbf{A}_{k}\right)^{\text {ét,Br }}=X\left(\mathbf{A}_{k}\right)^{G \text {-ét, } \operatorname{Br}_{G}}=X\left(\mathbf{A}_{k}\right)^{\mathrm{Br}_{G}(X)} .
$$

Ce résultat particulier peut s'établir aussi via l'approximation forte sur $X$ par rapport à $\operatorname{Br}_{G}(X)$ (voir [BD, Thm. 1.4]).

Donnons maintenant la structure de l'article.

$\mathrm{Au} \S 2$, sur un corps $k$ quelconque, s'inspirant de la notion de torseur universel de ColliotThélène et Sansuc, on introduit la notion de torseur universel de $n$-torsion (Déf. 2.1). Ensuite, on utilise cette notion à établir une formule de Künneth spéciale pour la cohomologie étale de degré 2 .

$\mathrm{Au} \S 3$, sur un corps $k$ de caractéristique zéro, on considère la donnée d'un $k$-groupe algébrique $G$, d'une $G$-variété $X$ lisse, d'un $k$-groupe fini $F$, d'un torseur $Y \rightarrow X$ sous $F$, on donne des conditions équivalentes pour le relèvement, de façon compatible, de l'action de $G$ sur $X$ en une action sur $Y$. Ce relèvement n'est pas toujours possible. On étudie les homomorphismes surjectifs de groupes algébriques connexes $H \rightarrow G$ avec une action compatible de $H$ sur $Y$, et on montre qu'il existe un objet minimal $H_{Y}$. Étant donné un élément $\alpha \in \operatorname{Br}(X)$, en utilisant la formule de Künneth ci-dessus, on montre ensuite qu'il existe un torseur $Y \rightarrow X$ sous un $k$-groupe fini commutatif $F$ tel que l'image réciproque de $\alpha$ dans $\operatorname{Br}(Y)$ soit invariante sous $H_{Y}$.

$\mathrm{Au} \S 4$, on rappelle des notions et des résultats établis dans [C18, §3], en particulier, la notion de sous-groupe de Brauer invariant et aussi ses propriétés élémentaires. Ces résultats seront utilisés dans les $\S 5$ et $\S 6$.

$\mathrm{Au} \S 5$, le corps de base $k$ est un corps de nombres. Dans [C18], étant donné un torseur $Y \rightarrow X$ sous un groupe linéaire connexe $G$, j'ai développé la méthode de descente des points adéliques orthogonaux aux sous-groupes de Brauer invariants. Au §5, on donne deux nouvelles variantes de cette descente. La première (Proposition 5.1) traite du cas où $G$ est un $k$-groupe fini commutatif. La seconde (Proposition 5.5) implique l'obstruction de Brauer-Manin étale invariante.

Les $\S 3, \S 4$ et $\S 5$ sont utilisés de façon essentielle au $\S 6$ où l'on établit le théorème 1.4.

$\mathrm{Au} \S 7$, en combinant le théorème 1.4 et la proposition 5.5 , on établit les théorèmes 1.1 et 1.2.

\section{Conventions et notations.}

Soit $k$ un corps quelconque de caractéristique char $(k)$. On note $\bar{k}$ une clôture algébrique, $k_{s}$ une clôture séparable et $\Gamma_{k}:=\operatorname{Gal}\left(k_{s} / k\right)$. Si $\operatorname{char}(k)=0$, on a $k_{s}=\bar{k}$ et $\Gamma_{k}:=\operatorname{Gal}(\bar{k} / k)$.

Tous les groupes de cohomologie sont des groupes de cohomologie étale.

Une $k$-variété $X$ est un $k$-schéma séparé de type fini. Pour $X$ une telle variété, on note $k[X]$ son anneau des fonctions globales, $k[X]^{\times}$son groupe des fonctions inversibles, $\operatorname{Pic}(X):=$ 
$H_{\text {êt }}^{1}\left(X, \mathbb{G}_{m}\right)$ son groupe de Picard et $\operatorname{Br}(X):=H_{\text {êt }}^{2}\left(X, \mathbb{G}_{m}\right)$ son groupe de Brauer. Notons

$$
\operatorname{Br}_{1}(X):=\operatorname{Ker}\left[\operatorname{Br}(X) \rightarrow \operatorname{Br}\left(X_{\bar{k}}\right)\right] \text { et } \operatorname{Br}_{a}(X):=\operatorname{Br}_{1}(X) / \operatorname{ImBr}(k) .
$$

Le groupe $\operatorname{Br}_{1}(X)$ est le sous-groupe "algébrique" du groupe de Brauer de $X$. Si $X$ est intègre, on note $k(X)$ son corps des fonctions rationnelles et $\pi_{1}(X, \bar{x})$ (ou $\left.\pi_{1}(X)\right)$ son groupe fondamental étale, où $\bar{x}$ est un point géométrique de $X$. Soit $\pi_{1}\left(X_{k_{s}}\right)^{a b}$ le quotient maximal abélien de $\pi_{1}\left(X_{k_{s}}\right)$. Alors $\pi_{1}\left(X_{k_{s}}\right)^{a b}$ est un $\Gamma_{k}$-module.

Un $k$-groupe algébrique $G$ est une $k$-variété qui est un $k$-schéma en groupes. On note $e_{G}$ l'unité de $G$ et $G^{*}:=\operatorname{Hom}_{k_{s} \text {-groupe }}\left(G_{k_{s}}, \mathbb{G}_{m}\right)$ le groupe des caractères de $G_{k_{s}}$. C'est un module galoisien de type fini. De plus, si $G$ est connexe sur $\mathbb{C}$, le groupe $\pi_{1}(G)$ est commutatif (cf. [BS, Prop. $1.1(2)]$ ).

Un $k$-groupe fini $F$ est un $k$-groupe algébrique qui est fini sur $k$. Dans ce cas, $F$ est déterminé par le $\Gamma_{k}$-groupe $F\left(k_{s}\right)$. Pour toute $k$-variété lisse connexe $X$, on a un isomorphisme canonique ([SGA1, §XI.5]) :

$$
H^{1}\left(\pi_{1}(X), F\left(k_{s}\right)\right) \stackrel{\sim}{\rightarrow} H^{1}(X, F) \quad \text { et donc } \quad H^{1}\left(X_{k_{s}}, F\right) \cong \operatorname{Hom}_{\text {cont }}\left(\pi_{1}\left(X_{k_{s}}\right), F\left(k_{s}\right)\right) / \sim
$$

où l'action de $\pi_{1}(X)$ sur $F\left(k_{s}\right)$ est induite par celle de $\Gamma_{k}$ et $\sim$ est induite par la conjugaison.

Soit $G$ un $k$-groupe algébrique. Une $G$-variété $(X, \rho)$ (ou $X$ ) est une $k$-variété $X$ munie d'une action à gauche $G \times_{k} X \stackrel{\rho}{\rightarrow} X$. Un $k$-morphisme de $G$-variétés est appelé $G$-morphisme s'il est compatible avec l'action de $G$.

Comme déjà indiqué ci-dessus, pour tout $k$-groupe algébrique $F$, tout $F$-torseur $f: Y \rightarrow X$ et tout $\sigma \in H^{1}(k, F)$, on note $F_{\sigma}$ (resp. $f_{\sigma}: Y_{\sigma} \rightarrow X$ ) le tordu de $F$ (resp. de $f$ ). Ainsi $f_{\sigma}$ est un $F_{\sigma}$-torseur.

\section{Torseur universel De N-TORSiOn et FORMule De KÜNneth DE DeGré 2}

Dans toute cette section, $k$ est un corps quelconque. Sauf mention explicite du contraire, une variété est une $k$-variété. Fixons un entier $n \geq 2$ avec $\operatorname{char}(k) \nmid n$ et notons $-\otimes-:=-\otimes_{\mathbb{Z} / n}-$.

Cette section contient deux parties. On introduit d'abord la version de $n$-torsion de la notion de torseur universel (Colliot-Thélène et Sansuc) dans la définition 2.1, et aussi la notion de type prolongé d'un torseur (Harari et Skorobogatov) dans la proposition 2.2. En utilisant ces notions, on considère ensuite le cup-produit de la cohomologie étale de degré 2 sur un produit de deux variétés quelconques et on établit une formule de Künneth pour ce produit (Prop. 2.6). Cette formule généralise un résultat de Skorobogatov et Zarhin, qui traite du cas où les deux variétés sont propres.

Soient $\operatorname{Sh}(k)$ la catégorie des faisceaux étales sur le petit site de Spec $k$ et $D^{+}(k)$ la catégorie dérivée bornée à gauche de $\operatorname{Sh}(k)$ et $D^{b}(k)$ la catégorie dérivée bornée de $\operatorname{Sh}(k)$ (une souscatégorie pleine de $\left.D^{+}(k)\right)$. Pour tout $i \in \mathbb{Z}$, on a les sous-catégories canoniques $D^{\geq i}(k)$ et $D^{\leq i}(k)$ de $D^{+}(k)$ et deux foncteurs canoniques $\tau_{\leq i}, \tau_{\geq i}$ ([KS, Déf. 12.3.1 et Prop. 13.1.5]). Donc $\operatorname{Sh}(k)=D^{\geq 0}(k) \cap D^{\leq 0}(k)$ est une sous-catégorie pleine canonique de $D^{+}(k)$. Par abus de notation, pour un objet $M$ de $\operatorname{Sh}(k)$, on note $M$ l'objet de $D^{+}(k)$ représenté par le complexe qui consiste en $M$ en degré 0.

Soient $X$ une variété géométriquement intègre et $p: X \rightarrow$ Spec $k$ le morphisme de structure. Soit $S_{X}$ un groupe de type multiplicatif tel que $S_{X}^{*} \cong H^{1}\left(X_{k_{s}}, \mu_{n}\right)$ comme $\Gamma_{k}$-modules. On rappelle que $H^{1}\left(X_{k_{s}}, \mu_{n}\right)$ est fini. 
Dans $D^{+}(k)$, il existe deux morphismes canoniques $\mathbb{G}_{m} \rightarrow R p_{*} \mathbb{G}_{m}$ et $\mu_{n} \rightarrow R p_{*} \mu_{n}$. Soient $\Delta$ le cône de $\mathbb{G}_{m}[1] \rightarrow R p_{*} \mathbb{G}_{m}[1]$ et $\Delta_{n}$ le cône de $\mu_{n}[1] \rightarrow R p_{*} \mu_{n}[1]$. La suite exacte de Kummer donne un diagramme commutatif de triangles distingués :

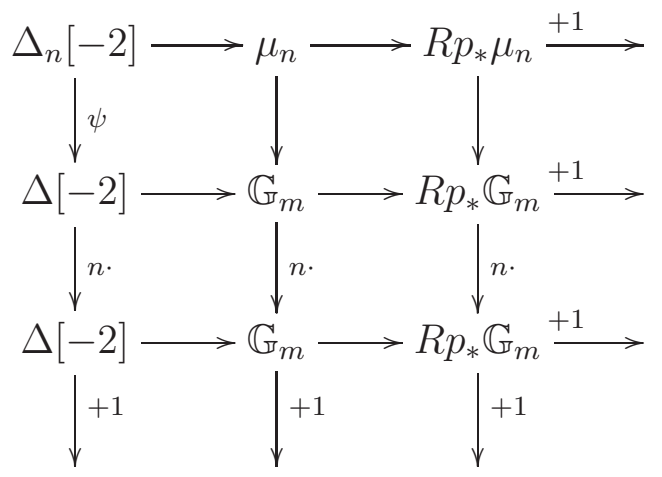

Les faisceaux de cohomologie des complexes $\Delta_{n}$ et $\Delta$ se calculent comme suit :

$$
\begin{gathered}
\Delta_{n} \in D^{\geq 0}(k), \quad \mathcal{H}^{0}\left(\Delta_{n}\right) \cong H^{1}\left(X_{k_{s}}, \mu_{n}\right) \cong S_{X}^{*}, \\
\Delta \in D^{\geq-1}(k), \quad \mathcal{H}^{-1}(\Delta)=k_{s}[X]^{\times} / k_{s}^{\times} \quad \text { et } \quad \mathcal{H}^{0}(\Delta)=\operatorname{Pic}\left(X_{k_{s}}\right) .
\end{gathered}
$$

Le morphisme $\psi: \Delta_{n} \rightarrow \Delta$ induit un morphisme $\psi_{\leq 0}:=\tau_{\leq 0} \psi: S_{X}^{*} \rightarrow \tau_{\leq 0} \Delta$.

Harari et Skorobogatov montrent que, pour tout groupe de type multiplicatif $S$, on a une suite exacte naturelle ([HS13, Prop. 1.1], où $\tau_{\leq 0} \Delta$ est noté $\left.K D^{\prime}(X)\right)$ :

$$
H^{1}(k, S) \rightarrow H^{1}(X, S) \stackrel{\chi}{\rightarrow} \operatorname{Hom}_{D^{+}(k)}\left(S^{*}, \tau_{\leq 0} \Delta\right) \rightarrow H^{2}(k, S) .
$$

Définition 2.1. Un torseur universel de $n$-torsion pour $X$ est un $S_{X}$-torseur $\mathcal{T}_{X}$ sur $X$ tel que $\chi\left(\left[\mathcal{T}_{X}\right]\right)=\psi_{\leq 0}: S_{X}^{*} \rightarrow \tau_{\leq 0} \Delta$.

D'après [HS13, Prop. 1.3], si $X(k) \neq \emptyset$, pour chaque $x \in X(k)$, il existe alors un unique torseur universel de $n$-torsion $\mathcal{T}_{X}$ pour $X$ tel que $x^{*}\left[\mathcal{T}_{X}\right]=0 \in H^{1}\left(k, S_{X}\right)$.

Dans le cas où $k$ est un corps de nombres, il existe un torseur universel de $n$-torsion pour $X$ lorsque $X\left(\mathbf{A}_{k}\right)^{\operatorname{Br}_{1}(X)} \neq \emptyset([\mathrm{HS} 13$, Cor. 3.6]).

Proposition 2.2. Soit $\mathcal{T}_{X}$ un torseur universel de $n$-torsion pour $X$. Soit $S$ un groupe de type multiplicatif tel que $n \cdot S=0$. Alors, pour tout $S$-torseur $Y$ sur $X$, il existe un unique homomorphisme $\phi: S_{X} \rightarrow S$ tel que

$$
\phi_{*}\left(\left[\mathcal{T}_{X}\right]\right)-[Y] \in \operatorname{Im}\left(H^{1}(k, S) \rightarrow H^{1}(X, S)\right) .
$$

Démonstration. Le triangle $\Delta_{n} \rightarrow \Delta \stackrel{n_{\dot{*}}}{\rightarrow} \Delta \stackrel{+1}{\rightarrow}$ induit une suite exacte

$$
\operatorname{Hom}_{D^{+}(k)}\left(S^{*}, \Delta[-1]\right) \rightarrow \operatorname{Hom}_{D^{+}(k)}\left(S^{*}, \Delta_{n}\right) \rightarrow \operatorname{Hom}_{D^{+}(k)}\left(S^{*}, \Delta\right) \stackrel{n \cdot}{\rightarrow} \operatorname{Hom}_{D^{+}(k)}\left(S^{*}, \Delta\right) .
$$

Puisque $S^{*} \in D^{\leq 0}(k)$ et $n \cdot S^{*}=0$, on a

$$
\operatorname{Hom}_{D^{+}(k)}\left(S^{*}, \Delta[-1]\right)=\operatorname{Hom}_{k}\left(S^{*}, \mathcal{H}^{-1}(\Delta)\right)=\operatorname{Hom}_{k}\left(S^{*}, \bar{k}[X]^{\times} / \bar{k}^{\times}\right)=0
$$

et donc $\operatorname{Hom}_{k}\left(S^{*}, S_{X}^{*}\right)$ est isomorphe à

$$
\operatorname{Hom}_{D^{+}(k)}\left(S^{*}, S_{X}^{*}\right) \stackrel{\sim}{\rightarrow} \operatorname{Hom}_{D^{+}(k)}\left(S^{*}, \Delta_{n}\right) \stackrel{\sim}{\rightarrow} \operatorname{Hom}_{D^{+}(k)}\left(S^{*}, \Delta\right) \stackrel{\sim}{\leftarrow} \operatorname{Hom}_{D^{+}(k)}\left(S^{*}, \tau_{\leq 0} \Delta\right) .
$$


Alors $\chi([Y]) \in \operatorname{Hom}_{D^{+}(k)}\left(S^{*}, \tau_{\leq 0} \Delta\right)$ donne un homomorphisme $\phi^{*} \in \operatorname{Hom}_{k}\left(S^{*}, S_{X}^{*}\right)$, et donc $\chi([Y])=\psi_{\leq 0} \circ \phi^{*}$. Soit $\phi: S_{X} \rightarrow S$ l'homomorphisme correspondant. La suite exacte (2.1) implique l'énoncé.

L'homomorphisme $\phi$ dans la proposition 2.2 est appelé le $n$-type de $[Y]$.

Soit $\mathcal{T}_{X}$ le torseur universel de $n$-torsion pour $X_{k_{s}}$, on obtient un isomorphisme de $\Gamma_{k^{-}}$ modules :

$$
\tau_{X, S}: \operatorname{Hom}_{k_{s}}\left(S_{X}, S\right) \cong \operatorname{Hom}_{k_{s}}\left(S^{*}, S_{X}^{*}\right) \rightarrow H^{1}\left(X_{k_{s}}, S\right): \phi \mapsto \phi_{*}\left(\left[\mathcal{T}_{X}\right]\right) .
$$

En particulier, on a deux $\Gamma_{k}$-isomorphismes naturels

$$
\tau_{X}:=\tau_{X, \mu_{n}}: S_{X}^{*} \stackrel{\sim}{\rightarrow} H^{1}\left(X_{k_{s}}, \mu_{n}\right): \phi \mapsto \phi_{*}\left(\mathcal{T}_{X}\right)
$$

et

$$
\tau_{X}(-1):=\tau_{X, \mathbb{Z} / n}: \operatorname{Hom}_{k_{s}}\left(S_{X}, \mathbb{Z} / n\right) \stackrel{\sim}{\rightarrow} H^{1}\left(X_{k_{s}}, \mathbb{Z} / n\right): \phi \mapsto \phi_{*}\left(\mathcal{T}_{X}\right) .
$$

En fait, par définition, $\tau_{X}$ est exactement l'homomorphisme $S_{X}^{*} \rightarrow \mathcal{H}^{0}\left(\Delta_{n}\right)$ induit par $\psi_{\leq 0}$.

Rappelons que, pour tous $F_{1}, F_{2} \in D^{b}(k)$, le produit tensoriel $F_{1} \otimes^{L} F_{2}$ est bien défini et le cup-produit est le homomorphisme canonique

$$
\cup_{j}: \oplus_{r+s=j} \mathcal{H}^{r}\left(F_{1}\right) \otimes \mathcal{H}^{s}\left(F_{2}\right) \rightarrow \mathcal{H}^{j}\left(F_{1} \otimes^{L} F_{2}\right)
$$

induit par la suite spectrale de Godement (cf. [Mi, Lem. VI. 8.6] ou [Fu, Prop. 6.4.12]). De plus, $R p_{*} \mu_{n} \in D^{b}(k)([\mathrm{Fu}$, Cor. 7.5.6]).

Corollaire 2.3. Supposons que $k$ est séparablement clos. Soit $p: X \rightarrow$ Spec $k$ une variété intègre. Alors

(1) le cup-produit $\cup: H^{1}\left(X, \mu_{n}\right) \otimes \operatorname{Hom}\left(\mu_{n}, S\right) \rightarrow H^{1}(X, S):(\alpha, \varphi) \mapsto \varphi_{*}(\alpha)$ est un isomorphisme;

(2) pour tout complexe de $\mathbb{Z} / n$-modules (vus comme $k$-faisceaux) de type fini $F$ avec $F \in$ $D^{\geq 0}(k) \cap D^{b}(k)$, on a $R p_{*} \mu_{n} \otimes^{L} F \in D^{\geq 0}(k)$ et le cup-produit

$$
\cup_{j}(F): \oplus_{r+s=j} R^{r} p_{*} \mu_{n} \otimes \mathcal{H}^{s}(F) \cong \oplus_{r+s=j} \mathcal{H}^{r}\left(R p_{*} \mu_{n}\right) \otimes \mathcal{H}^{s}(F) \rightarrow \mathcal{H}^{j}\left(R p_{*} \mu_{n} \otimes^{L} F\right)
$$

est un isomorphisme pour $j=0$ et $j=1$;

(3) dans (2), si $\mathcal{H}^{0}(F)$ est plat, alors $\cup_{2}(F)$ est un isomorphisme.

Démonstration. Puisque $X(k) \neq \emptyset$, il existe un torseur universel de $n$-torsion $\mathcal{T}_{X} \rightarrow X$. D'après (2.2), on a le diagramme

$$
\begin{gathered}
\operatorname{Hom}\left(S_{X}, \mu_{n}\right) \otimes \operatorname{Hom}\left(\mu_{n}, S\right) \stackrel{\stackrel{-\circ-}{\longrightarrow} \operatorname{Hom}\left(S_{X}, S\right)}{\cong} \quad \cong \tau_{X, S} \\
\cong \tau_{X} \otimes i d \\
H^{1}\left(X, \mu_{n}\right) \otimes \operatorname{Hom}\left(\mu_{n}, S\right) \stackrel{\cup}{\longrightarrow} H^{1}(X, S),
\end{gathered}
$$

où $-\circ-:(\psi, \phi) \mapsto \phi \circ \psi$. Ce diagramme est commutatif car

$$
\tau_{X, S}(\varphi \circ \phi)=(\varphi \circ \phi)_{*}\left[\mathcal{T}_{X}\right]=\varphi_{*}\left(\phi_{*}\left[\mathcal{T}_{X}\right]\right) \stackrel{(2.3)}{=} \tau_{X}[\phi] \cup \varphi
$$

pour tout $\phi \in \operatorname{Hom}\left(S_{X}, \mu_{n}\right)$ et tout $\varphi \in \operatorname{Hom}\left(\mu_{n}, S\right)$. Donc on a (1).

Pour tout complexe $F$ dans (2), puisque la dimension cohomologique de $R p_{*}$ est finie ([SGA4, XIV], cf. [Fu, Cor. 7.5.6]), on a : 
(i) d'après [SGA4, XVII. Thm. 5.2.11], pour tout $j<0$, on a $\mathcal{H}^{j}\left(R p_{*} \mu_{n} \otimes^{L} F\right)=0$ et donc $R p_{*} \mu_{n} \otimes^{L} F \in D^{\geq 0}(k)$. Ceci implique le premier énoncé de $(2)$;

(ii) si $F \cong \mathcal{H}^{0}(F)$ avec $\mathcal{H}^{0}(F)$ plat, on a $\mathcal{H}^{j}\left(R p_{*} \mu_{n} \otimes^{L} F\right)=\mathcal{H}^{j}\left(R p_{*} \mu_{n}\right) \otimes F$ et donc $\cup_{j}(F)$ est un isomorphisme pour tout $j$;

(iii) si $F \cong \mathcal{H}^{0}(F)$, on a $R p_{*} \mu_{n} \otimes^{L} F \cong R p_{*}\left(\mu_{n} \otimes p^{*} F\right)$ ([SGA4, XVII. (5.2.11.1)], cf. [Fu, Cor. 6.5.6]). Puisque $X$ est intègre, $\cup_{0}(F): \mu_{n} \otimes F \rightarrow R^{0} p_{*}\left(\mu_{n} \otimes p^{*} F\right)$ est un isomorphisme, et d'après l'énoncé (1) et [Mi, Prop. V.1.20], le cup-produit

$\cup_{1}(F): R^{1} p_{*} \mu_{n} \otimes F \cong H^{1}\left(X, \mu_{n}\right) \otimes \operatorname{Hom}\left(\mu_{n}, \mu_{n} \otimes F\right) \stackrel{\cup}{\rightarrow} H^{1}\left(X, \mu_{n} \otimes F\right) \cong \mathcal{H}^{1}\left(R p_{*}\left(\mu_{n} \otimes p^{*} F\right)\right)$ est un isomorphisme. Ceci vaut seulement pour le cup-produit de degré 1.

Pour tout complexe $F$ dans $(2)$, notons $F_{+}:=\left(\tau_{\geq 1} F\right)[1]$ un objet dans $D^{\geq 0}(k) \cap D^{b}(k)$. Alors $F_{+}$vérifie toutes les hypothèses dans $(2)$.

Le triangle $\mathcal{H}^{0}(F) \rightarrow F \rightarrow F_{+}[-1] \stackrel{+1}{\longrightarrow}$ donne un diagramme commutatif de suites exactes :

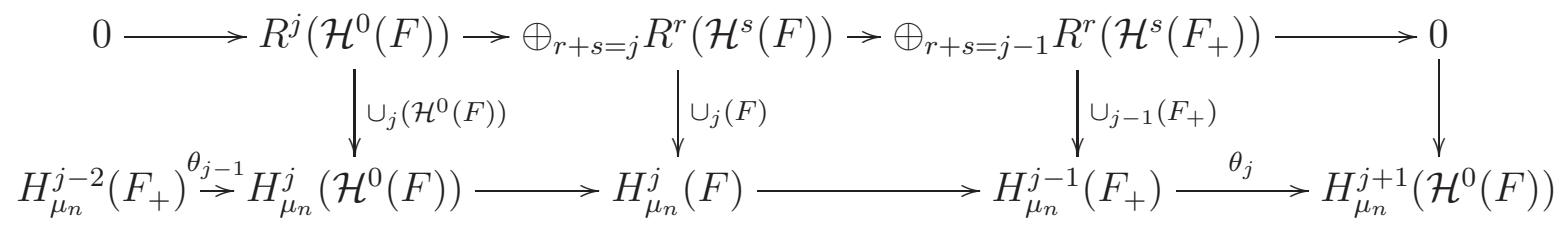

où $H_{\mu_{n}}^{j}(-):=\mathcal{H}^{j}\left(R p_{*} \mu_{n} \otimes^{L}-\right), R^{r}(-):=R^{r} p_{*} \mu_{n} \otimes-$ et la première ligne est exacte car elle est scindée.

Montrons l'énoncé (2). D'après (i), on a : $H_{\mu_{n}}^{-2}\left(F_{+}\right)=H_{\mu_{n}}^{-1}\left(F_{+}\right)=0$. D'après (iii), $\cup_{0}\left(\mathcal{H}^{0}(F)\right)$ est un isomorphisme. Le lemme des cinq implique : $\cup_{0}(F)$ est un isomorphisme. Ceci donne l'énoncé(2) pour $j=0$. Donc $\cup_{0}\left(F_{+}\right)$est un isomorphisme. D'après (iii), $\cup_{1}\left(\mathcal{H}^{0}(F)\right)$ est un isomorphisme. Le lemme des cinq implique : $\cup_{1}(F)$ est un isomorphisme.

Montrons l'énoncé (3). Par hypothèse, $\mathcal{H}^{0}(F)$ est plat, et d'après (ii), $\cup_{2}\left(\mathcal{H}^{0}(F)\right)$ est un isomorphisme. D'après $(2), \cup_{1}\left(F_{+}\right)$et $\cup_{0}\left(F_{+}\right)$sont des isomorphismes. Donc $\theta_{1}=0$ et le lemme des cinq implique : $\cup_{2}(F)$ est un isomorphisme.

Si $X$ est lisse, d'après (1.5), l'isomorphisme (2.4) donne un $\Gamma_{k}$-isomorphisme naturel

$$
\tau_{X}(-1): \operatorname{Hom}\left(S_{X}, \mathbb{Z} / n\right) \stackrel{\sim}{\rightarrow} H^{1}\left(X_{k_{s}}, \mathbb{Z} / n\right) \cong \operatorname{Hom}_{\text {cont }}\left(\pi_{1}\left(X_{k_{s}}\right)^{a b}, \mathbb{Z} / n\right): \psi \mapsto \psi\left(k_{s}\right) \circ \tau_{\pi_{1}},
$$

où $\tau_{\pi_{1}}: \pi_{1}\left(X_{k_{s}}\right)^{a b} \rightarrow S_{X}\left(k_{s}\right)$ est l'homomorphisme induit par $\mathcal{T}_{X}$. Ainsi $\tau_{\pi_{1}}$ induit un isomorphisme de $\Gamma_{k}$-modules $\pi_{1}\left(X_{k_{s}}\right)^{a b} / n \stackrel{\sim}{\rightarrow} S_{X}\left(k_{s}\right)$ et $\mathcal{T}_{X}$ est géométriquement intègre.

Corollaire 2.4. Soit $X$ une variété lisse géométriquement intègre. Soient $M$ un $\mathbb{Z} / n$-module et $\pi_{1}\left(X_{k_{s}}\right) \stackrel{\theta}{\rightarrow} M$ un homomorphisme surjectif de noyau $\Gamma_{k}$-invariant. Supposons qu'il existe un torseur universel de $n$-torsion pour $X$. Alors il existe un $k$-groupe fini commutatif $S$ et un $S$-torseur $\mathcal{T} \rightarrow X$ tels que $\mathcal{T}$ soit lisse géométriquement intègre, $S\left(k_{s}\right)=M$ et que, dans $H^{1}\left(X_{k_{s}}, S\right) \cong \operatorname{Hom}_{\text {cont }}\left(\pi_{1}\left(X_{k_{s}}\right)^{a b}, M\right)$, on ait $\left[\mathcal{T}_{k_{s}}\right]=\theta$.

Démonstration. Soit $\mathcal{T}_{X}$ un torseur universel de $n$-torsion pour $X$ (un torseur sous le $k$-groupe $\left.S_{X}\right)$. Puisque $\operatorname{Ker}(\theta)$ est $\Gamma_{k}$-invariant, il existe une unique $\Gamma_{k}$-structure sur $M$ telle que $\theta$ soit un $\Gamma_{k}$-morphisme. Ceci induit un $k$-groupe commutatif $S$ et un homomorphisme surjectif $\theta^{\prime}: S_{X} \rightarrow S$ tels que $S\left(k_{s}\right)=M$ et que $\theta^{\prime}\left(k_{s}\right) \circ \tau_{\pi_{1}}=\theta$. Alors $\mathcal{T}:=\theta_{*}^{\prime} \mathcal{T}_{X}:=\mathcal{T}_{X} \times{ }^{S_{X}} S$ donne l'énoncé. 
Soient $U, V$ deux variétés géométriquement intègres sur $k$. On considère le diagramme commutatif

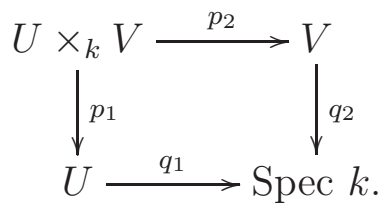

Soient $M, N$ deux $\mathbb{Z} / n$-faisceaux finis plats sur le grand site de $k$. Le cup-produit donne un quasi-isomorphisme ([SGA $4 \frac{1}{2}$, Th. finitude Cor. 1.11], cf. [Fu, Cor. 9.3.5]) :

$$
\cup: R q_{1, *} M \otimes^{L} R q_{2, *} N \cong R\left(q_{1} \circ p_{1}\right)_{*}\left(M \otimes^{L} N\right) .
$$

Ceci induit le cup-produit ([Fu, Prop. 6.4.12]) :

$$
\cup_{j}: \oplus_{r+s=j} R^{r} q_{1, *} M \otimes_{\mathbb{Z} / n} R^{s} q_{2, *} N \rightarrow \mathcal{H}^{j}\left(R q_{1, *} M \otimes^{L} R q_{2, *} N\right) \stackrel{\sim}{\rightarrow} R^{j}\left(q_{1} \circ p_{1}\right)_{*}\left(M \otimes^{L} N\right) .
$$

Lemme 2.5. Le cup-produit $\cup_{j}$ est un isomorphisme pour $j=0,1,2$.

Démonstration. On peut supposer que $k$ est séparablement clos. Les $\mathbb{Z} / n$-modules finis $M$, $N$ sont plats et donc ils sont des facteurs directs de $(\mathbb{Z} / n)^{\oplus i}$ pour $i$ assez grand. Puisque tous les foncteurs ci-dessus commutent avec les sommes directs finies, on peut supposer que $M=N=\mu_{n}$. L'énoncé découle du corollaire 2.3 (3) et de (2.8).

Le résultat ci-dessous généralise [SZ, Thm. 2.6].

Proposition 2.6. Supposons que $k$ est séparablement clos. Soient $U, V$ deux variétés géométriquement intègres et $F$ un $\mathbb{Z} / n$-module fini plat. On considère le diagramme (2.7). Alors on a des isomorphismes naturels:

$$
\left(p_{1}^{*}, p_{2}^{*}\right): H^{1}(U, F) \oplus H^{1}(V, F) \stackrel{\sim}{\rightarrow} H^{1}(U \times V, F)
$$

et

$$
\left(p_{1}^{*}, \cup, p_{2}^{*}\right): H^{2}(U, F) \oplus\left[H^{1}(U, \mathbb{Z} / n) \otimes_{\mathbb{Z}} H^{1}(V, F)\right] \oplus H^{2}(V, F) \stackrel{\sim}{\rightarrow} H^{2}(U \times V, F),
$$
ò̀ $\cup: H^{1}(U, \mathbb{Z} / n) \otimes_{\mathbb{Z}} H^{1}(V, F) \rightarrow H^{2}(U \times V, F)$ est le cup-produit.

C'est clair que si $U, V$ sont définis sur un sous-corps $k_{0} \subset k$ avec $k / k_{0}$ galoisienne et $F$ un $\operatorname{Gal}\left(k / k_{0}\right)$-module, alors les deux isomorphismes ci-dessus sont des isomorphismes de $\operatorname{Gal}\left(k / k_{0}\right)$ modules.

Si $\operatorname{char}(k)=0$, cette proposition découle de [SZ, Prop. 2.2] et de [Mi, Thm. III.3.12] (on peut vérifier que l'homomorphisme dans [SZ, Prop. 2.2] est compactible avec le cup-produit).

Démonstration. On applique le lemme 2.5 au cas $U \times k$ et au cas $k \times V$, et on obtient deux diagrammes commutatifs :

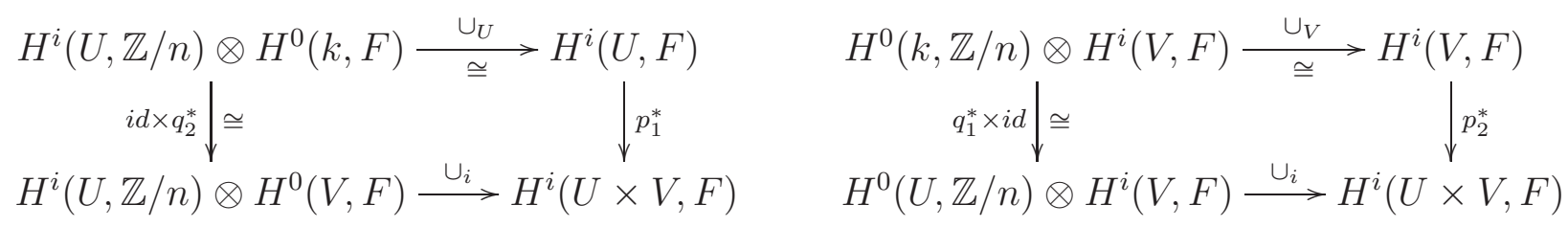

pour $i=1$ et 2 , où $\cup_{U}$ (resp. $\cup_{V}$ ) est le cup-produit sur $U$ (resp. $V$ ). Donc $p_{1}^{*}\left(H^{i}(U, F)\right)=\cup_{i}\left(H^{i}(U, \mathbb{Z} / n) \otimes H^{0}(V, F)\right) \quad$ et $\quad p_{2}^{*}\left(H^{i}(V, F)\right)=\cup_{i}\left(H^{0}(U, \mathbb{Z} / n) \otimes H^{i}(V, F)\right)$.

L'énoncé découle du lemme 2.5. 
Soient $\mathcal{T}_{U}$ (resp. $\mathcal{T}_{V}$ ) un torseur universel de $n$-torsion pour $U$ (resp. pour $V$ ) et $S_{U}$ (resp. $S_{V}$ ) le groupe correspondant (cf. Définition 2.1). Skorobogatov et Zarhin introduisent un homomorphisme ([SZ, §5]) :

$$
\varepsilon: \operatorname{Hom}_{k}\left(S_{U}, S_{V}^{*}\right) \rightarrow H^{2}\left(U \times V, \mu_{n}\right): \phi \mapsto \phi_{*}\left[\mathcal{T}_{U}\right] \cup\left[\mathcal{T}_{V}\right],
$$

où $\cup$ est le cup-produit $H^{1}\left(U, S_{V}^{*}\right) \times H^{1}\left(V, S_{V}\right) \rightarrow H^{2}\left(U \times V, \mu_{n}\right)$. Les isomorphismes $\tau_{V}$ dans (2.3) et $\tau_{U}(-1)$ dans (2.4) donnent un diagramme :

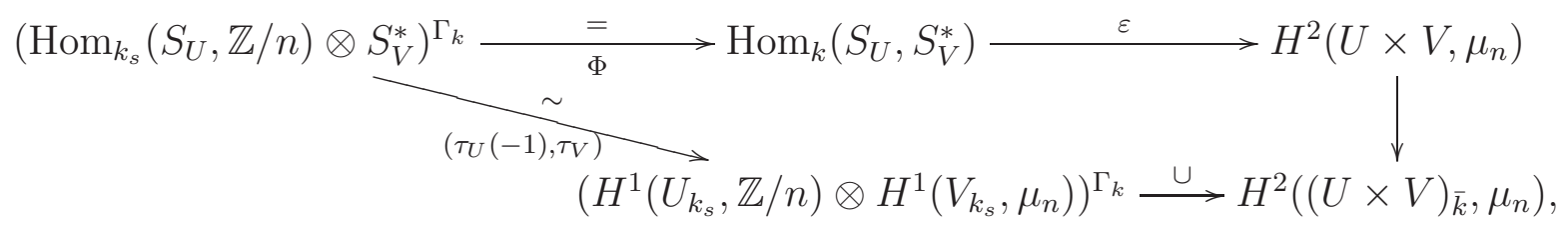

qui est commutatif parce que, pour tous $\varphi \in \operatorname{Hom}_{k_{s}}\left(S_{U}, \mathbb{Z} / n\right)$ et $\phi \in S_{V}^{*}=\operatorname{Hom}_{k_{s}}\left(S_{V}, \mu_{n}\right)$, on note $\phi^{*}:=\operatorname{Hom}_{k_{s}}\left(\phi, \mu_{n}\right): \mathbb{Z} / n \rightarrow S_{V}^{*}$ le dual de $\phi$, et on a :

$$
\varepsilon(\Phi(\varphi \otimes \phi))=\varepsilon\left(\phi^{*} \circ \varphi\right)=\left(\phi^{*}\right)_{*}\left(\varphi_{*}\left[\mathcal{T}_{U}\right]\right) \cup\left[\mathcal{T}_{V}\right] \stackrel{(1)}{=} \varphi_{*}\left[\mathcal{T}_{U}\right] \cup \phi_{*}\left[\mathcal{T}_{V}\right]=\tau_{U}(-1)(\varphi) \cup \tau_{V}(\phi),
$$

où (1) découle du diagramme commutatif

$$
\begin{aligned}
& H^{1}\left(U \times V, S_{V}\right) \times H^{1}\left(U \times V, \operatorname{Hom}_{k_{s}}\left(S_{V}, \mu_{n}\right)\right) \stackrel{\cup}{\longrightarrow} H^{2}\left(U \times V, \mu_{n}\right) \\
& \phi_{*} \quad\left(\phi^{*}\right)_{*}=\operatorname{Hom}_{k_{s}}\left(\phi, \mu_{n}\right)_{*} \uparrow \quad \downarrow= \\
& H^{1}\left(U \times V, \mu_{n}\right) \times H^{1}\left(U \times V, \operatorname{Hom}_{k_{s}}\left(\mu_{n}, \mu_{n}\right)\right) \stackrel{\cup}{\longrightarrow} H^{2}\left(U \times V, \mu_{n}\right) .
\end{aligned}
$$

Si $U(k) \neq \emptyset$, alors il existe un torseur universel de $n$-torsion pour $U$. Pour un point $u \in U(k)$, notons

$$
H_{u}^{i}\left(U, \mu_{n}\right):=\operatorname{Ker}\left(H^{i}\left(U, \mu_{n}\right) \stackrel{u^{*}}{\rightarrow} H^{i}\left(k, \mu_{n}\right)\right) .
$$

Corollaire 2.7. Sous les notations et hypothèses ci-dessus, supposons que $U(k) \neq \emptyset$ avec $u \in$ $U(k)$ et qu'il existe des torseurs universels de n-torsion $\mathcal{T}_{U}$ pour $U$ (sous le groupe $S_{U}$ ) et $\mathcal{T}_{V}$ pour $V$ (sous le groupe $S_{V}$ ). Alors on a un isomorphisme:

$$
H_{u}^{2}\left(U, \mu_{n}\right) \oplus H^{2}\left(V, \mu_{n}\right) \oplus \operatorname{Hom}_{k}\left(S_{U}, S_{V}^{*}\right) \stackrel{\left(p_{1}^{*}, p_{2}^{*}, \varepsilon\right)}{\longrightarrow} H^{2}\left(U \times V, \mu_{n}\right) .
$$

Démonstration. Notons $E_{2}^{i, j}(U):=H^{i}\left(k, H^{j}\left(U_{k_{s}}, \mu_{n}\right)\right) \Rightarrow H^{i+j}\left(U, \mu_{n}\right)$ la suite spectrale de Hochschild-Serre de $U$ et $E_{2}^{i, j}(V)$ (resp. $E_{2}^{i, j}(U \times V)$ ) celle de $V$ (resp. de $\left.U \times V\right)$.

Notons $H_{u}^{i}\left(U_{k_{s}}, \mu_{n}\right):=\operatorname{Ker}\left(H^{i}\left(U_{k_{s}}, \mu_{n}\right) \stackrel{u^{*}}{\rightarrow} H^{i}\left(k_{s}, \mu_{n}\right)\right)$. Alors $H_{u}^{0}\left(U_{k_{s}}, \mu_{n}\right)=0$ et $H_{u}^{i}\left(U_{k_{s}}, \mu_{n}\right)=$ $H^{i}\left(U_{k_{s}}, \mu_{n}\right)$ pour $i \neq 0$. La suite spectrale de Hochschild-Serre donne canoniquement une suite spectrale :

$$
E_{2}^{i, j}(U, u):=H^{i}\left(k, H_{u}^{j}\left(U_{k_{s}}, \mu_{n}\right)\right) \Rightarrow H_{u}^{i+j}\left(U, \mu_{n}\right) .
$$

Soit $\phi_{2}^{i, j}: E_{2}^{i, j}(U, u) \oplus E_{2}^{i, j}(V) \rightarrow E_{2}^{i, j}(U \times V)$ le morphisme de suites spectrales induit par $\left(p_{1}^{*}, p_{2}^{*}\right)$. D'après la proposition $2.6, \phi_{2}^{i, j}$ est un isomorphisme pour $j=0,1$ et $\phi_{2}^{0,2}$ est injectif. Ainsi $\phi_{2}^{i, j}$ induit une suite exacte par le lemme des cinq :

$$
0 \rightarrow H_{u}^{2}\left(U, \mu_{n}\right) \oplus H^{2}\left(V, \mu_{n}\right) \stackrel{p_{1}^{*}, p_{2}^{*}}{\longrightarrow} H^{2}\left(U \times V, \mu_{n}\right) \rightarrow \operatorname{coker}\left(\phi_{2}^{0,2}\right) .
$$


D'après la proposition 2.6 et le diagramme (2.10), on a coker $\left(\phi_{2}^{0,2}\right) \cong\left(H^{1}\left(U_{\bar{k}}, \mathbb{Z} / n\right) \otimes H^{1}\left(V_{\bar{k}}, \mu_{n}\right)\right)^{\Gamma_{k}}$ et la composition

$$
\operatorname{Hom}_{k}\left(S_{U}, S_{V}^{*}\right) \stackrel{\varepsilon}{\rightarrow} H^{2}\left(U \times V, \mu_{n}\right) \rightarrow H^{2}\left((U \times V)_{\bar{k}}, \mu_{n}\right)^{\Gamma_{k}} \rightarrow \operatorname{coker}\left(\phi_{2}^{0,2}\right)
$$

est un isomorphisme, d'où le résultat.

\section{Préliminaires sur les torseurs sous un Groupe Fini}

Dans toute cette section, $k$ est un corps quelconque de caractéristique 0. Sauf mention explicite du contraire, une variété est une $k$-variété.

Soit $G$ un groupe algébrique connexe et $X$ une $G$-variété lisse géométriquement intègre. Cette section traite trois problèmes : pour un torseur $H \rightarrow G$ sous un $k$-groupe fini, on montre l'existence et l'unicité de la structure de groupe sur $H$ dans $\S 3.1$; pour un torseur $Y \rightarrow X$ sous un $k$-groupe fini, on donne dans $§ 3.2$ une condition nécessaire et suffisante pour le relèvement, de façon compatible, de l'action de $G$ sur $X$ en une action sur $Y$; si ce relèvement n'existe pas, on montre dans $\S 3.3$ l'existence d'une isogénie minimale $H_{Y} \rightarrow G$ telle que l'action de $H_{Y}$ puisse être relevée en une action sur $Y$.

3.1. Torseur sur un groupe algébrique. Pour un groupe algébrique connexe $G$, tout recouvrement étale fini de $G_{\bar{k}}$ est une extension centrale de $G_{\bar{k}}$ (Brion et Szamuely [BS, Prop. 1.1 (1)]). Le résultat suivant généralise ce résultat au corps de base et il est aussi un analogue d'un résultat de Colliot-Thélène ([CT08, Thm. 5.6]).

Proposition 3.1. Soit $G$ un groupe algébrique connexe, $S$ un k-groupe fini commutatif et $\psi$ : $H \rightarrow G$ un $S$-torseur avec $H$ géométriquement intègre sur $k$. S'il existe un point $e_{H} \in H(k)$ avec $\psi\left(e_{H}\right)=e_{G}$, alors il existe une unique structure de $k$-groupe algébrique sur $H$ telle que $\psi$ soit un homomorphisme et que $e_{H}$ soit l'unité.

De plus, dans ce cas, $\operatorname{Ker}(\psi)=S$ et l'action de $S$ sur $H$ est compatible avec la multiplication de $H$.

Démonstration. L'existence d'une structure de groupe sur $H$ est équivalente à l'existence d'un couple de morphismes $\left(m_{H}, i_{H}\right)$ satisfaisant certaines relations où $m_{H}: H \times H \rightarrow H$ est la multiplication et $i_{H}: H \rightarrow H$ est l'inverse.

Pour l'unicité, s'il existe deux structures de groupe sur $H$, soient $\left(m_{H}, i_{H}\right),\left(m_{H}^{\prime}, i_{H}^{\prime}\right)$ les couples de morphismes correspondants. Soient $m_{G}$ la multiplication de $G$ et $i_{G}$ l'inverse de $G$. Alors

$$
\psi \circ m_{H}=m_{G} \circ(\psi \times \psi)=\psi \circ m_{H}^{\prime}, \quad m_{H}\left(e_{H} \times e_{H}\right)=e_{H}=m_{H}^{\prime}\left(e_{H} \times e_{H}\right)
$$

et

$$
\psi \circ i_{H}=i_{G} \circ \psi=\psi \circ i_{H}^{\prime}, \quad i_{H}\left(e_{H}\right)=e_{H}=i_{H}^{\prime}\left(e_{H}\right) .
$$

Puisque $\psi$ est fini étale et $H \times H$ est intègre, on a $m_{H}=m_{H}^{\prime}$ et $i_{H}=i_{H}^{\prime}$ ([Mi, Cor. I.3.13]). Ceci donne l'unicité de $\left(m_{H}, i_{H}\right)$.

Pour l'existence de la structure de groupe (i.e. l'existence de $\left.\left(m_{H}, i_{H}\right)\right)$, par la descente galoisienne et l'unicité de $\left(m_{H}, i_{H}\right)$, il suffit d'établir l'existence de $\left(m_{H}, i_{H}\right)$ sur $\bar{k}$. On peut supposer que $k=\bar{k}$. Dans ce cas, $\psi$ est fini étale galoisien avec $\operatorname{Aut}(H / G) \cong S(\bar{k})$. D'après [BS, Prop. 1.1 (1)], il existe une structure de groupe sur $H$ telle que $\psi: H \rightarrow G$ soit une isogénie centrale. Notons $-\cdot-$ la multiplication et $(-)^{-1}$ l'inverse de cette structure de groupe. Soit $c:=e_{H} \cdot e_{H}$ et $d:=e_{H} \cdot c^{-1}$. Les points $e_{H}, c$ et $d$ sont dans $\operatorname{Ker}(\psi)$ et donc dans le centre de 
$H$. Alors les morphismes $m_{H}^{\prime}: H \times H \rightarrow H:\left(h_{1}, h_{2}\right) \mapsto d \cdot h_{1} \cdot h_{2}$ et $i_{H}^{\prime}: H \rightarrow H: h \mapsto c \cdot h^{-1}$ définissent sur $H$ une nouvelle structure de groupe et cette structure vérifie les hypothèses ci-dessus.

Pour le dernier énoncé, puisque $S \subset \operatorname{Ker}(\psi)$, l'action de $S$ induit une inclusion de $\Gamma_{k}$-module $S(\bar{k}) \subset \operatorname{Aut}\left(H_{\bar{k}} / G_{\bar{k}}\right)$ et la multiplication de $H$ induit une inclusion $\operatorname{Ker}(\psi)(\bar{k}) \subset \operatorname{Aut}\left(H_{\bar{k}} / G_{\bar{k}}\right)$ de $\Gamma_{k}$-module. Puisque \#Aut $\left(H_{\bar{k}} / G_{\bar{k}}\right)=\operatorname{deg}(\psi)$, les deux inclusions ci-dessus sont isomorphes, d'où le résultat.

Corollaire 3.2. Soit $G$ un groupe algébrique connexe. Pour tout $\mathbb{Z} / n$-module fini $M$ et tout homomorphisme surjectif $\pi_{1}\left(G_{\bar{k}}\right) \stackrel{\theta}{\rightarrow} M$ de noyau $\Gamma_{k}$-invariant, il existe un unique groupe algébrique connexe $H$ isogène à $G$, i.e. muni d'un homomorphisme fini surjectif $\psi: H \rightarrow G$, tel que $(\operatorname{Ker}(\psi))(\bar{k}) \cong M$ et que la composition $\pi_{1}\left(H_{\bar{k}}\right) \stackrel{\psi_{\pi_{1}}}{\rightarrow} \pi_{1}\left(G_{\bar{k}}\right) \stackrel{\theta}{\rightarrow} M$ soit nulle.

De plus, pour tout groupe algébrique connexe $H_{1}$, tout homomorphisme fini surjectif $\psi_{1}$ : $H_{1} \rightarrow G$ vérifiant $\theta \circ \psi_{1, \pi_{1}}=0$ se factorise par $\psi$.

Démonstration. Puisque $G(k) \neq \emptyset$, il existe un unique torseur universel de $n$-torsion $\mathcal{T}_{G}$ (un $S_{G}$-torseur sur $G$ ) tel que $\left.\mathcal{T}_{G}\right|_{e_{G}} \cong S_{G}$.

D'après le corollaire 2.4, il existe un $k$-groupe fini commutatif $S$ et un $S$-torseur $H \stackrel{\psi}{\rightarrow} G$ tels que $S(\bar{k})=M$ et que l'homomorphisme $\pi_{1}\left(G_{\bar{k}}\right) \rightarrow S(\bar{k})$ induit par $\left[H_{\bar{k}}\right]$ soit $\theta$. Donc la composition $\theta \circ \psi_{\pi_{1}}$ est nulle. Après avoir tordu par un élément de $H^{1}(k, S)$, on peut supposer que $\left.[H]\right|_{e_{G}}=0 \in H^{1}(k, S)$. D'après la proposition 3.1, il existe une structure de groupe sur $H$ telle que $\psi$ soit un homomorphisme et que $\operatorname{Ker}(\psi)=S$.

Pour tout groupe algébrique connexe $H_{1}$ et tout homomorphisme fini surjectif $\psi_{1}: H_{1} \rightarrow G$, le noyau $\psi_{1}$ est commutatif et on a une suite exacte de $\Gamma_{k}$-modules :

$$
\pi_{1}\left(H_{1, \bar{k}}\right) \rightarrow \pi_{1}\left(G_{\bar{k}}\right) \rightarrow \operatorname{Ker}\left(\psi_{1}\right)(\bar{k}) \rightarrow 0 .
$$

Ceci donne un homomorphisme surjectif de $\Gamma_{k}$-modules $\theta_{1}: \operatorname{Ker}\left(\psi_{1}\right)(\bar{k}) \rightarrow M \cong S(\bar{k})$ et, puisque $\left.\left[H_{1}\right]\right|_{e_{G}}=0=\left.[H]\right|_{e_{G}}$, on a $\theta_{1, *}\left(\left[H_{1}\right]\right)=[H] \in H^{1}(X, S)$. En utilisant l'action de $S$, on a un $\operatorname{Ker}\left(\psi_{1}\right)$-morphisme $\phi: H_{1} \rightarrow H$ au-dessus de $G$ tel que $\phi\left(e_{H_{1}}\right)=e_{H}$. Soient $\chi_{1}, \chi_{2}: H_{1} \times H_{1} \rightarrow H$ deux morphismes avec $\chi_{1}\left(h_{1}, h_{2}\right)=\phi\left(h_{1} \cdot h_{2}\right)$ et $\chi_{2}\left(h_{1}, h_{2}\right)=\phi\left(h_{1}\right) \cdot \phi\left(h_{2}\right)$ pour tous $h_{1}, h_{2} \in H_{1}$. Alors $\chi_{1}\left(e_{H_{1}}, e_{H_{1}}\right)=\chi_{2}\left(e_{H_{1}}, e_{H_{1}}\right)$ et $\psi \circ \chi_{1}=\psi \circ \chi_{2}$. Ceci induit :

$$
\chi: H_{1} \times_{k} H_{1} \stackrel{\chi_{1}, \chi_{2}}{\longrightarrow} H \times_{G} H \cong H \times_{k} S \stackrel{p_{2}}{\longrightarrow} S .
$$

Puisque $H_{1}$ est connexe, on a $\operatorname{Im}(\chi)=e_{S}, \chi_{1}=\chi_{2}$ et $\phi$ est un homomorphisme.

3.2. Relèvement d'une action par un torseur. Soient $G$ un groupe algébrique connexe et $(X, \rho)$ une $G$-variété lisse géométriquement intègre. Soient $F$ un $k$-groupe fini et $f: Y \rightarrow X$ un $F$-torseur. Notons $p_{1}: G \times X \rightarrow G, p_{2}: G \times X \rightarrow X$ les deux projections.

D'après [SGA1, X.2.2], on a deux suites exactes de groupes fondamentaux

$$
1 \rightarrow \pi_{1}\left(X_{\bar{k}}\right) \rightarrow \pi_{1}(X) \rightarrow \Gamma_{k} \rightarrow 1 \quad \text { et } \quad 1 \rightarrow \pi_{1}\left((G \times X)_{\bar{k}}\right) \rightarrow \pi_{1}(G \times X) \rightarrow \Gamma_{k} \rightarrow 1 .
$$

D'après [SGA1, XII.5.2], on a $\pi_{1}\left((G \times X)_{\bar{k}}\right) \cong \pi_{1}\left(G_{\bar{k}}\right) \times \pi_{1}\left(X_{\bar{k}}\right)$, car ceci vaut pour les espaces topologiques. Alors on a une suite exacte de groupes fondamentaux :

$$
1 \rightarrow \pi_{1}\left(G_{\bar{k}}\right) \rightarrow \pi_{1}(G \times X) \stackrel{p_{2, \pi_{1}}}{\longrightarrow} \pi_{1}(X) \rightarrow 1
$$


qui admet une section induite par $i_{e}: X \rightarrow G \times X: x \mapsto\left(e_{G}, x\right)$ et l'action de $\pi_{1}(X)$ sur $\pi_{1}\left(G_{\bar{k}}\right)$ se factorise par $\Gamma_{k}$. D'après (1.5), cette suite exacte induit une suite exacte d'ensembles pointés (voir $[$ Se65, §5.8])

$$
1 \rightarrow H^{1}(X, F) \stackrel{p_{2}^{*}}{\rightarrow} H^{1}(G \times X, F) \stackrel{\iota}{\rightarrow} H^{1}\left(G_{\bar{k}}, F\right)^{\Gamma_{k}}
$$

et $p_{2}^{*}$ admet une section induite par $i_{e}^{*}$.

Proposition 3.3. Soient $G$ un groupe algébrique connexe et $(X, \rho)$ une $G$-variété lisse géométriquement intègre. Soient $F$ un k-groupe fini et $f: Y \rightarrow X$ un F-torseur. Alors les hypothèses ci-dessous sont équivalentes :

(a) on a $\rho^{*}([Y])=p_{2}^{*}([Y]) \in H^{1}(G \times X, F)$;

(b) pour $\iota$ dans (3.1), on a $\iota\left(\rho^{*}([Y])\right)=0 \in H^{1}\left(G_{\bar{k}}, F\right)$;

(c) le $F$-torseur $Y$ est $G$-compatible, i.e. l'action de $G$ sur $X$ se relève en une action sur $Y$;

(d) il existe un morphisme $\rho_{Y}: G \times Y \rightarrow Y$ tel que $\left.\rho_{Y}\right|_{e_{G} \times Y}=i d_{Y}$ et que $\rho_{Y}$ soit compatible avec $\rho$, i.e. $\rho \circ\left(i d_{G} \times f\right)=f \circ \rho_{Y}$.

De plus, sous les hypothèses ci-dessus, on a

(1) l'action de $G$ sur $Y$ pour laquelle $f$ est un $G$-morphisme est unique;

(2) l'action de $G$ et celle de $F$ commutent;

(3) pour tout $\sigma \in H^{1}(k, F)$, le $F_{\sigma}$-torseur $Y_{\sigma}$ est $G$-compatible.

Démonstration. Puisque $i_{e}^{*}\left(p_{2}^{*}([Y])\right)=i_{e}^{*}\left(\rho^{*}([Y])\right)$ dans $H^{1}(X, F)$, l'équivalence $(\mathrm{a}) \Leftrightarrow(\mathrm{b})$ découle de la suite exacte (3.1).

Lemme 3.4. Pour tout $k$-schéma de type fini $Z$ et tous morphismes $\theta_{1}, \theta_{2}: G \times Z \rightarrow Y$, si $f \circ \theta_{1}=f \circ \theta_{2}$ et $\left.\theta_{1}\right|_{e_{G} \times Z}=\left.\theta_{2}\right|_{e_{G} \times Z}$, alors $\theta_{1}=\theta_{2}$.

Démonstration. En fait, $\theta_{1}, \theta_{2}$ induisent un morphisme

$$
\theta: G \times Z \stackrel{\left(\theta_{1}, \theta_{2}\right)}{\longrightarrow} Y \times_{X} Y \cong Y \times_{k} F \stackrel{p r_{F}}{\longrightarrow} F
$$

tel que $\theta\left(e_{G} \times Z\right)=e_{F}$. Puisque $G$ est intègre, $\theta(G \times Z)=e_{F}$ et donc $\theta_{1}=\theta_{2}$.

Pour $(\mathrm{a}) \Rightarrow(\mathrm{d})$, soient $\rho^{*} Y$ le pullback de $Y$ par $\rho$ et $p_{2}^{*} Y:=G \times Y$. Notons $\operatorname{Mor}_{F}\left(p_{2}^{*} Y, F\right)$ l'ensemble des morphismes $\chi: p_{2}^{*} Y \rightarrow F$ tels que $\chi(a \cdot y)=a \cdot \chi(y) \cdot a^{-1}$ pour tous $a \in F$ et $y \in$ $p_{2}^{*} Y$. Définissons de même $\operatorname{Mor}_{F}(Y, F)$. Alors $Y \cong e_{G} \times Y \subset p_{2}^{*} Y$ induit un morphisme surjectif $\operatorname{Mor}_{F}\left(p_{2}^{*} Y, F\right) \stackrel{\operatorname{Mor}\left(i_{e}\right)}{\longrightarrow} \operatorname{Mor}_{F}(Y, F)$, car il existe une section induite par $p_{2}$. Par hypothèse, on a un isomorphisme de $F$-torseur $p_{2}^{*} Y \stackrel{\phi}{\rightarrow} \rho^{*} Y$. Pour tout isomorphisme $\phi_{1}$, l'argument classique montre qu'il existe un $\chi_{1} \in \operatorname{Mor}_{F}\left(p_{2}^{*} Y, F\right)$ tel que $\phi_{1}=\chi_{1} \cdot \phi$. Puisque $\operatorname{Mor}\left(i_{e}\right)$ est surjectif, on peut supposer que $\left.\phi\right|_{e_{G} \times X}$ est l'identité de $Y$. Le morphisme $\rho_{Y}: G \times Y \stackrel{\phi}{\rightarrow} \rho^{*} Y \rightarrow Y$ donne (d).

Pour $(\mathrm{d}) \Rightarrow(\mathrm{c})$, l'hypothèse $(\mathrm{d})$ donne un diagramme commutatif :

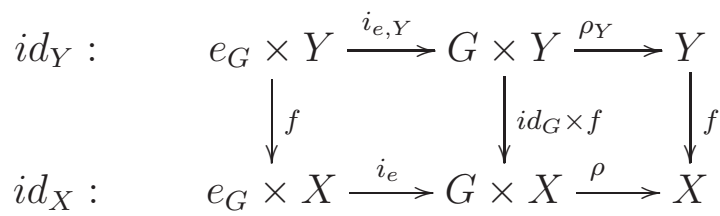

tel que $\rho_{Y} \circ i_{e, Y}=i d_{Y}$. Soient $\theta_{1}, \theta_{2}: G \times G \times Y \rightarrow Y$ les deux morphismes définis par

$$
\theta_{1}\left(g_{1}, g_{2}, y\right)=g_{1} \cdot\left(g_{2} \cdot y\right) \quad \text { et } \quad \theta_{2}\left(g_{1}, g_{2}, y\right)=\left(g_{1} \cdot g_{2}\right) \cdot y
$$


pour tous $g_{1}, g_{2} \in G$ et $y \in Y$. Alors $\theta_{1}\left(e_{G}, g_{2}, y\right)=\theta_{2}\left(e_{G}, g_{2}, y\right)$ et le lemme 3.4 montre que $\theta_{1}=\theta_{2}$. Donc $\rho_{Y}$ est une action et $f$ est un $G$-morphisme. Ceci donne (c).

Supposons (c) et montrons (1), (2), (3) et (a). $Y$.

L'hypothèse (c) donne aussi le diagramme commutatif (3.2) avec $\rho_{Y}$ l'action relevée de $G$ sur

Soient $\theta_{1}, \theta_{2}$ deux actions de $G$ sur $Y$ telles que $f$ soit un $G$-morphisme. Puisque $f \circ \theta_{1}=$ $\rho \circ\left(i d_{G} \times f\right)=f \circ \theta_{2}$, On applique le lemme 3.4 à $\theta_{1}, \theta_{2}: G \times Y \rightarrow Y$ et on obtient (1).

Soient $\theta_{1}, \theta_{2}: G \times F \times Y \rightarrow Y$ les deux morphismes définis par $\theta_{1}(g, a, y)=g \cdot(a \cdot y)$ et $\theta_{2}(g, a, y)=a \cdot(g \cdot y)$ pour tous $g \in G, a \in F$ et $y \in Y$. Alors $\theta_{1}\left(e_{G}, a, y\right)=a \cdot y=\theta_{2}\left(e_{G}, a, y\right)$ et $\left(f \circ \theta_{1}\right)(g, a, y)=g \cdot f(y)=\left(f \circ \theta_{2}\right)(g, a, y)$. On applique le lemme 3.4 à $\theta_{1}, \theta_{2}: G \times F \times Y \rightarrow Y$ et on obtient (2).

Pour le $F$-torseur $p_{2}^{*}([Y])=(G \times Y \rightarrow G \times X)$, l'énoncé (2) montre que l'action $G \times Y \rightarrow Y$ est un $F$-morphisme compatible avec $\rho$. Ceci induit un isomorphisme de $F$-torseurs $p_{2}^{*}([Y])=$ $\rho^{*}([Y])$ et on a $(\mathrm{a})$.

Puisque l'énoncé (b) est un énoncé sur $\bar{k}$, on obtient (3).

Corollaire 3.5. Soient $G$ un groupe algébrique connexe et $(X, \rho)$ une $G$-variété lisse géométriquement intègre. Alors $\rho$ induit un homomorphisme $\rho_{\pi_{1}}: \pi_{1}\left(G_{\bar{k}}\right) \rightarrow \pi_{1}(X)$ et, pour tout $k$-groupe fini $F$, il induit $\rho_{\pi_{1}}^{*}: H^{1}(X, F) \rightarrow H^{1}\left(G_{\bar{k}}, F\right)$ et on a :

(1) le sous-groupe $\operatorname{Im}\left(\rho_{\pi_{1}}\right) \subset \pi_{1}(X)$ est normal et il est contenu dans le centre de $\pi_{1}\left(X_{\bar{k}}\right)$;

(2) pour tout $\alpha \in H^{1}(X, F)$, on a $\rho_{\pi_{1}}^{*}(\alpha)=\iota\left(\rho^{*}(\alpha)\right)$, où ı est dans (3.1);

(3) pour tout 1-cocycle a de $\pi_{1}(X)$ à valeurs dans $F(\bar{k})$, l'homomorphisme a $\circ \rho_{\pi_{1}}: \pi_{1}\left(G_{\bar{k}}\right) \rightarrow$ $F(\bar{k})$ est de noyau $\Gamma_{k}$-invariant, et il est nul si et seulement si $\rho_{\pi_{1}}^{*}([a])=0$;

(4) si $X$ est un $G$-espace homogène à stabilisateur géométrique connexe, alors tout $F$-torseur $G$-compatible est constant, i.e. ce torseur est isomorphe à $M \times_{k} X$ avec $M$ un F-torseur sur $k$.

Démonstration. L'énoncé (1) vaut car $\pi_{1}\left(G_{\bar{k}}\right)=\operatorname{Ker}\left(\pi_{1}(G \times X) \stackrel{p_{2, *}}{\longrightarrow} \pi_{1}(X)\right)$ et $\pi_{1}\left((G \times X)_{\bar{k}}\right) \cong$ $\pi_{1}\left(G_{\bar{k}}\right) \times \pi_{1}\left(X_{\bar{k}}\right)$. Les énoncés (2) et (3) découlent par définition.

Pour (4), dans ce cas, $\operatorname{Im}\left(\rho_{\pi_{1}}\right)=\pi_{1}\left(X_{\bar{k}}\right)$ ([Sz, Prop. 5.5.4]). D'après la proposition 3.3 et $(2)$, (3) ci-dessus, tout $F$-torseur $G$-compatible est trivial sur $X_{\bar{k}}$, et donc il provient d'un $F$-torseur sur $k$.

Corollaire 3.6. Sous les notations et les hypothèses ci-dessus, supposons que $f$ est G-compatible. Alors, pour tout $k$-schéma fini étale $E$, la restriction de Weil $V:=R_{X \times E / X}(Y \times E)$ est un $R_{E / k}\left(F \times{ }_{k} E\right)$-torseur $G$-compatible sur $X$.

Démonstration. Notons $f_{V}: V \rightarrow X$. Par hypothèse, $f_{V}$ est un torseur sous le groupe

$$
R_{X \times E / X}(F \times X \times E) \cong R_{E / k}\left(F \times_{k} E\right) .
$$

On considère $G \times V$ comme un $X$-schéma par le morphisme $G \times V \stackrel{i d_{G} \times f_{V}}{\rightarrow} G \times X \stackrel{\rho}{\rightarrow} X$ et $G \times Y$ comme un $X$-schéma par $\rho \circ\left(i d_{G} \times f\right)$. Dans ce cas, tout morphisme $\rho_{V} \in \operatorname{Mor}_{X}(G \times V, V)$ satisfait $f_{V} \circ \rho_{V}=\rho \circ\left(i d_{G} \times f_{V}\right)$. D'après la proposition $3.3(\mathrm{~d})$, il suffit de trouver un $\rho_{V} \in$ $\operatorname{Mor}_{X}(G \times V, V)$ tel que $\left.\rho_{V}\right|_{e_{G} \times V}=i d_{V}$. Puisque

$\operatorname{Mor}_{X}(V, V) \stackrel{\sim}{\rightarrow} \operatorname{Mor}_{X \times E}(V \times E, Y \times E)$ et que $\operatorname{Mor}_{X}(G \times V, V) \stackrel{\sim}{\rightarrow} \operatorname{Mor}_{X \times E}(G \times V \times E, Y \times E)$, 
l'identité $i d_{V}$ induit un morphisme $V \times E \stackrel{\theta}{\rightarrow} Y \times E$. Le $X \times E$-morphisme

$$
G \times V \times E \stackrel{i d_{G} \times \theta}{\longrightarrow} G \times Y \times E \stackrel{\rho_{Y} \times i d_{E}}{\longrightarrow} Y \times E
$$

induit un morphisme $\rho_{V} \in \operatorname{Mor}_{X}(G \times V, V)$ qui satisfait $\left.\rho_{V}\right|_{e_{G} \times V}=i d_{V}$.

Corollaire 3.7. Soient $G$ un groupe algébrique connexe, $Z$ une variété lisse géométriquement intègre et $p: X \rightarrow Z$ un $G$-torseur. Pour tout $k$-groupe fini $F$ et tout $F$-torseur $G$-compatible $Y \rightarrow X$, il existe un F-torseur $Y_{Z}$ sur $Z$ tel que $[Y]=p^{*}\left(\left[Y_{Z}\right]\right) \in H^{1}(X, F)$.

Démonstration. D'après la proposition $3.3(2), Y$ est un $G \times F$-torseur sur $Z$ tel que $Y / F=X$. Alors $Y_{Z}:=Y / G$ est un $F$-torseur sur $Z$ et $Y \rightarrow Y_{Z}$ est un $F$-morphisme. Donc $[Y]=$ $p^{*}\left(\left[Y_{Z}\right]\right)$.

3.3. Le groupe minimal compatible avec un torseur. Soit $G$ un groupe algébrique connexe. Soit $\mathcal{C}_{G}$ la catégorie des groupes algébriques connexes $H$ isogènes à $G$, i.e. munis d'un homomorphisme fini surjectif $\psi: H \rightarrow G$. C'est clair que si $G$ est linéaire, tout objet dans $\mathcal{C}_{G}$ est aussi linéaire.

Soit $(X, \rho)$ une $G$-variété lisse géométriquement intègre. Soient $F$ un $k$-groupe fini et $f: Y \rightarrow$ $X$ un $F$-torseur. Soit $\mathcal{C}_{G}(Y)$ la sous-catégorie pleine de $\mathcal{C}_{G}$ dont les objets sont les groupes $H$ isogènes à $G$ tels que $f$ soit $H$-compatible. D'après la proposition $3.3(1)$, tout objet $H \in \mathcal{C}_{G}(Y)$ admet une unique action sur $Y$ telle que $f$ soit un $H$-morphisme. Alors tout morphisme de $\mathcal{C}_{G}(Y)$ est compatible avec les actions ci-dessus.

Proposition 3.8. La catégorie $\mathcal{C}_{G}(Y)$ admet un objet final $\left(H_{Y} \stackrel{\psi_{Y}}{\rightarrow} G\right)$, et un objet $(H \stackrel{\psi}{\rightarrow} G) \in$ $\mathcal{C}_{G}(Y)$ est final si et seulement si l'action de $\operatorname{ker}(\psi)$ sur $Y$ est libre.

Démonstration. Dans la suite exacte (3.1), notons $\alpha:=\iota\left(\rho^{*}([Y])\right) \in H^{1}\left(G_{\bar{k}}, F\right)^{\pi_{1}(X)}$. Soit $\theta \in$ $\operatorname{Hom}_{\text {cont }}\left(\pi_{1}\left(G_{\bar{k}}\right), F(\bar{k})\right)$ un élément correspondant à $\alpha$ selon (1.5). D'après le corollaire 3.5 (3), le noyau $\operatorname{Ker}(\theta)$ est $\Gamma_{k}$-invariant.

La fonctorialité de $(3.1)$ et la proposition 3.3 montrent qu'un objet $(H \stackrel{\psi}{\rightarrow} G) \in \mathcal{C}_{G}$ est contenu dans $\mathcal{C}_{G}(Y)$ si et seulement si $\psi_{*}(\alpha)=0 \in H^{1}\left(H_{\bar{k}}, F\right)$, i.e. $\theta \circ \psi_{\pi_{1}}=0$ (Corollaire 3.5 $(3)$ ), où $\psi_{\pi_{1}}: \pi_{1}\left(H_{\bar{k}}\right) \rightarrow \pi_{1}\left(G_{\bar{k}}\right)$. Puisque $\pi_{1}\left(G_{\bar{k}}\right)$ est abélien ([Miy, Thm. 1]), le corollaire 3.2 implique l'existence de l'objet final de $\mathcal{C}_{G}(Y)$.

L'argument ci-dessus montre que la catégorie $\mathcal{C}_{G}(Y)$ est stable par changement de base, i.e., pour toute $G$-variété $X^{\prime}$ et tout $G$-morphisme $X^{\prime} \rightarrow X$, on a un $F$-torseur $Y^{\prime}:=Y \times_{X} X^{\prime} \rightarrow X^{\prime}$ et $\mathcal{C}_{G}\left(Y^{\prime}\right)=\mathcal{C}_{G}(Y)$ comme sous-catégories de $\mathcal{C}_{G}$.

Soit $\left(H_{Y} \stackrel{\psi_{Y}}{\longrightarrow} G\right)$ l'objet final de $\mathcal{C}_{G}(Y)$. Il est l'objet final de $\mathcal{C}_{G}\left(Y^{\prime}\right)$ aussi pour tout $Y^{\prime} \rightarrow X^{\prime}$ ci-dessus.

Pour montrer que l'action de $\operatorname{Ker}\left(\psi_{Y}\right)$ est libre, on peut supposer que $k=\bar{k}$ et que $X$ est un espace homogène de $G$. Dans ce cas, $Y$ est un espace homogène de $F \times H_{Y}$ (Proposition 3.3 (2)). Puisque $\operatorname{Ker}\left(\psi_{Y}\right)$ est dans le centre de $F \times H_{Y}$, les stabilisateurs de $\operatorname{Ker}\left(\psi_{Y}\right)$ en tous les points $x \in X$ sont les mêmes. La propriété de l'objet final implique que l'action de $\operatorname{Ker}\left(\psi_{Y}\right)$ soit libre.

Soit $(H \stackrel{\psi}{\rightarrow} G) \in \mathcal{C}_{G}(Y)$ un objet tel que l'action de $\operatorname{ker}(\psi)$ sur $Y$ est libre. Soit $\phi: H \rightarrow$ $H_{Y}$ l'homomorphisme canonique. Puisque $\psi_{Y}, \psi$ sont finis surjectifs et que $H_{Y}$ est connexe, l'homomorphisme $\phi$ est fini surjectif. La proposition 3.3 (1) implique que $\phi$ est compatible avec l'action de $H$ et de $H_{Y}$. Puisque l'action de $\operatorname{Ker}(\psi)$ sur $Y$ est libre, $\phi$ est un isomorphisme. 
Définition 3.9. L'objet final $\left(H_{Y} \stackrel{\psi_{Y}}{\longrightarrow} G\right)$ de $\mathcal{C}_{G}(Y)$ est appelé le groupe minimal compatible avec le F-torseur $Y$.

Remarque 3.10. Soit $\rho_{\pi_{1}}: \pi_{1}\left(G_{\bar{k}}\right) \rightarrow \pi_{1}(X)$ l'homomorphisme dans le corollaire 3.5 et soit $\alpha$ un 1-cocycle de $\pi_{1}(X)$ en $F(\bar{k})$ qui correspond à $[Y] \in H^{1}(X, F)$. Alors $\left.\alpha\right|_{\pi_{1}\left(X_{\bar{k}}\right)}$ est un homomorphisme. Par la démonstration de la proposition 3.8, le groupe minimal compatible au $F$-torseur $Y$ est déterminé par $\operatorname{Ker}\left(\alpha \circ \rho_{\pi_{1}}\right)$, où $\alpha \circ \rho_{\pi_{1}}: \pi_{1}\left(G_{\bar{k}}\right) \rightarrow F(\bar{k})$ est un homomorphisme. Donc ceci est déterminé par $\operatorname{Ker}\left(\left.\alpha\right|_{\operatorname{Im}\left(\rho_{\pi_{1}}\right)}\right)$.

D'après la proposition $3.3(3), H_{Y}$ est aussi le groupe minimal compatible au $F_{\sigma}$-torseur $Y_{\sigma}$ pour tout $\sigma \in H^{1}(k, F)$.

Corollaire 3.11. Sous les notations et les hypothèses ci-dessus, si $Y$ est géométriquement intègre sur $k$, alors il existe un homomorphisme injectif $\phi: \operatorname{Ker}\left(\psi_{Y}\right) \rightarrow F$ d'image centrale compatible avec l'action de $\operatorname{Ker}\left(\psi_{Y}\right)$ et de $F$ sur $Y$.

Démonstration. L'action de $\operatorname{Ker}\left(\psi_{Y}\right)$ induit un morphisme :

$$
\Phi: \operatorname{Ker}\left(\psi_{Y}\right) \times Y \stackrel{\rho_{H_{Y}}, p r_{Y}}{\longrightarrow} Y \times_{X} Y \stackrel{\sim}{\rightarrow} F \times_{k} Y \stackrel{p r_{F}}{\longrightarrow} F,
$$

où $\rho_{H_{Y}}$ est l'action de $H_{Y}$. Pour tous $h \in \operatorname{Ker}\left(\psi_{Y}\right), y \in Y$, on a $h \cdot y=\Phi(h, y) \cdot y$.

Puisque $Y$ est géométriquement intègre, il existe un morphisme $\phi: \operatorname{Ker}\left(\psi_{Y}\right) \rightarrow F$ tel que $\Phi=\phi \circ p_{1}$, où $p_{1}: \operatorname{Ker}\left(\psi_{Y}\right) \times Y \rightarrow \operatorname{Ker}\left(\psi_{Y}\right)$ est la projection. Puisque l'action de $F$ sur $Y$ est libre, $\phi$ est un homomorphisme. La proposition 3.3 (2) implique que l'image de $\phi$ est centrale. D'après la proposition 3.8, l'action de $\operatorname{Ker}\left(\psi_{Y}\right)$ est libre et donc $\phi$ est injectif.

Rappelons la définition de $\operatorname{Br}_{G}(X)$ dans la définition 1.3.

Proposition 3.12. Soient $G$ un groupe algébrique connexe et $X$ une G-variété lisse géométriquement intègre. Supposons qu'il existe un torseur universel de $n$-torsion $\mathcal{T}_{X} \stackrel{f}{\rightarrow} X$ sous le groupe $S_{X}$. Soit $H \stackrel{\psi}{\rightarrow} G$ le groupe minimal compatible au $S_{X}$-torseur $\mathcal{T}_{X}$. Alors, pour tout élément de $n$-torsion $\alpha \in \operatorname{Br}(X)$ et tout $\sigma \in H^{1}\left(k, S_{X}\right)$, on a $f_{\sigma}^{*}(\alpha) \in \operatorname{Br}_{H}\left(\mathcal{T}_{X, \sigma}\right)$, où $f_{\sigma}^{*}$ : $\operatorname{Br}(X) \rightarrow \operatorname{Br}\left(\mathcal{T}_{X, \sigma}\right)$ est l'homomorphisme induit par $f_{\sigma}: \mathcal{T}_{X, \sigma} \rightarrow X$.

Démonstration. On peut supposer que $\sigma=0 \in H^{1}\left(k, S_{X}\right)$.

Notons $\rho_{H}: H \times \mathcal{T}_{X} \rightarrow \mathcal{T}_{X}$ l'action de $H$ et $p_{1, H}: H \times \mathcal{T}_{X} \rightarrow H, p_{2, H}: H \times \mathcal{T}_{X} \rightarrow \mathcal{T}_{X}$ les deux projections. Soit $\mathcal{T}_{G}$ un torseur universel de $n$-torsion pour $G$ sous le groupe $S_{G}$.

Apppliquant le corollaire 2.7 à $(G, X)$, on obtient : pour tout $\alpha_{1} \in H^{2}\left(X, \mu_{n}\right)$, il existe un $\phi \in \operatorname{Hom}\left(S_{G}, S_{X}^{*}\right)$ et un $\beta \in H^{2}\left(G, \mu_{n}\right)$ tels que $\left(\rho^{*}-p_{2}^{*}\right)\left(\alpha_{1}\right)=\varepsilon(\phi)+p_{1}^{*}(\beta)$.

Puisque $f^{*}\left(\left[\mathcal{T}_{X}\right]\right)=0 \in H^{1}\left(\mathcal{T}_{X}, S_{X}\right)$, on a

$$
(\psi \times f)^{*}(\varepsilon(\phi))=(\psi \times f)^{*}\left(\phi_{*}\left(\left[\mathcal{T}_{G}\right]\right) \cup\left[\mathcal{T}_{X}\right]\right)=\phi_{*}\left(\psi^{*}\left(\left[\mathcal{T}_{G}\right]\right)\right) \cup f^{*}\left(\left[\mathcal{T}_{X}\right]\right)=0 .
$$

Alors $\left(\rho_{H}^{*}-p_{2, H}^{*}\right)\left(f^{*}\left(\alpha_{1}\right)\right)=(\psi \times f)^{*}\left(\left(\rho^{*}-p_{2}^{*}\right)\left(\alpha_{1}\right)\right)=(\psi \times f)^{*}\left(p_{1}^{*}(\beta)\right)=p_{1, H}^{*}\left(\psi^{*}(\beta)\right)$.

D'après la suite exacte de Kummer, $\left(\rho_{H}^{*}-p_{2, H}^{*}\right)\left(f^{*}(\alpha)\right) \subset p_{1, H}^{*} \operatorname{Br}(H)$, d'où le résultat.

\section{RAPpel Sur LE SOUS-GRoupe DE BRAUER INVARIANT}

Dans toute cette section, $k$ est un corps quelconque de caractéristique 0. Sauf mention explicite du contraire, une variété est une $k$-variété. 
Dans cette section, on rappelle des notions et des résultats dans [C18, §3] sur le sous-groupe de Brauer invariant.

Pour la définition du sous-groupe de Brauer invariant on renvoie le lecteur à la définition 1.3.

Soit AB la catégorie des groupes abéliens. Soit GX la catégorie des couples $(G, X)$ avec $G$ un groupe algébrique connexe et $X$ une $G$-variété lisse, et un morphisme $(H, Y) \rightarrow(G, X)$ dans GX est un couple $(\psi, f)$ avec $\psi: H \rightarrow G$ un homomorphisme et $f: Y \rightarrow X$ un $H$-morphisme, où l'action de $H$ sur $X$ est induite par $\psi$. Par définition,

$$
\mathrm{Br}_{-}(-): \mathbf{G X} \rightarrow \mathbf{A B}:(G, X) \mapsto \mathrm{Br}_{G}(X)
$$

est un foncteur contravariant.

Exemple 4.1. (1) ([C18, Lem. 3.6]) Soit $G$ un groupe linéaire connexe. Alors $\operatorname{Br}_{G}(G)=\operatorname{Br}_{1}(G)$.

(2) ([C18, Prop. $3.9(3)])$ Soient $G$ un groupe linéaire connexe, $G_{0} \subset G$ un sous-groupe fermé connexe et $X:=G / G_{0}$. Alors

$$
\operatorname{Br}_{G}(X)=\operatorname{Br}_{1}(X, G):=\operatorname{ker}\left(\operatorname{Br}(X) \rightarrow \operatorname{Br}\left(G_{\bar{k}}\right)\right) .
$$

Le groupe $\operatorname{Br}_{1}(X, G)$ est défini par Borovoi et Demarche dans [BD] pour étudier l'approximation forte de $X$.

(3) Soit $A$ une variété abélienne. L'auteur ne sait pas identifier le groupe $\operatorname{Br}_{A}(A)$. Par exemple, l'auteur ne sait pas si $\operatorname{Br}_{A}(A) \subset \operatorname{Br}_{1}(A)$ ou si $\operatorname{Br}_{A}(A) \supset \operatorname{Br}_{1}(A)$.

Au vu de l'exemple 4.1 (3), dans la suite du présent article, on suppose que $G$ est un groupe linéaire.

Soient $G$ un groupe linéaire connexe et $X$ une $G$-variété lisse géométriquement intègre. Notons $\rho: G \times X \rightarrow X$ l'action et $p_{1}: G \times X \rightarrow G, p_{2}: G \times X \rightarrow X$ les deux projections.

(1) Puisque $\mathrm{Br}_{1}(G \times X) \cong \operatorname{Br}_{e}(G) \oplus \operatorname{Br}_{1}(X)$ ([S, Lem. 6.6]), on peut obtenir facilement ([C18, Prop. $3.2(4)])$ :

$$
\operatorname{Br}_{1}(X) \subset \operatorname{Br}_{G}(X)
$$

(2) Puisque $\left.p_{1}^{*}\right|_{\operatorname{Br}_{e}(G)}: \operatorname{Br}_{e}(G) \rightarrow \operatorname{Br}(G \times X)$ est injectif, par la définition de $\operatorname{Br}_{G}(X)$, il existe un unique homomorphisme $\operatorname{Br}_{G}(X) \stackrel{\lambda}{\rightarrow} \operatorname{Br}_{e}(G)$ tel que $([\mathrm{C} 18,(3.4)])$

$$
p_{1}^{*} \circ \lambda=\rho^{*}-p_{2}^{*}: \operatorname{Br}_{G}(X) \rightarrow \operatorname{Br}(G \times X) .
$$

Le $\lambda: \operatorname{Br}_{G}(X) \rightarrow \operatorname{Br}_{e}(G)$ est appelé l'homomorphisme de Sansuc ([C18, Déf. 3.8]).

(3) Pour toute extension de corps $K / k$, et tous $x \in X(K), g \in G(K), \alpha \in \mathrm{Br}_{G}(X)$, on a ([C18, Prop. $3.9(1)])$ :

$$
(g \cdot x)^{*}(\alpha)=g^{*}(\lambda(\alpha))+x^{*}(\alpha) \in \operatorname{Br}(K) .
$$

Alors, dans le cas où $k$ est un corps de nombres, on a :

$$
G\left(\mathbf{A}_{k}\right)^{\operatorname{Br}_{a}(G)} \cdot X\left(\mathbf{A}_{k}\right)^{\operatorname{Br}_{G}(X)}=X\left(\mathbf{A}_{k}\right)^{\operatorname{Br}_{G}(X)} .
$$

La formule (4.1) et [HS13, Cor. 3.6] impliquent directement :

Corollaire 4.2. Soit $(G, X) \in \mathbf{G X}$ un objet. Si $X\left(\mathbf{A}_{k}\right)^{\operatorname{Br}_{G}(X)} \neq \emptyset$, alors, pour tout entier $n \geq 2$, il existe un un torseur universel de $n$-torsion pour $X$.

Pour un torseur sous un groupe linéaire connexe, Sansuc a construit une suite exacte dans [S, Prop. 6.10], qui est appelée la suite exacte de Sansuc. La proposition suivante dit que les sous-groupes de Brauer invariant sont compatibles avec la suite exacte de Sansuc. 
Proposition 4.3. ([C18, Cor. 3.11(2)]) Soit $1 \rightarrow N \rightarrow H \stackrel{\psi}{\rightarrow} G \rightarrow 1$ une suite exacte de groupes linéaires connexes. Soit $(\psi, f):(H, Y) \rightarrow(G, X)$ un morphisme dans $\mathbf{G X}$ tel que $X$ soit géométriquement intègre sur $k$ et $Y \rightarrow X$ soit un $N$-torseur, où l'action de $N$ sur $Y$ est induite par celle de $H$. Alors $f^{*}: \operatorname{Br}(X) \rightarrow \operatorname{Br}(Y)$ satisfait $\left(f^{*}\right)^{-1} \operatorname{Br}_{H}(Y)=\operatorname{Br}_{G}(X)$ et on a une suite exacte, fonctorielle en $(X, Y, f, N)$ :

$$
\operatorname{Pic}(Y) \rightarrow \operatorname{Pic}(N) \rightarrow \operatorname{Br}_{G}(X) \stackrel{f^{*}}{\rightarrow} \operatorname{Br}_{H}(Y) \stackrel{\lambda}{\rightarrow} \operatorname{Br}_{e}(N)
$$

où $\lambda: \operatorname{Br}_{H}(Y) \subset \operatorname{Br}_{N}(Y) \rightarrow \operatorname{Br}_{e}(N)$ est l'homomorphisme de Sansuc.

Pour une fibration $f: X \rightarrow T$ et tout $t \in T(k)$, on note $i_{t}: X_{t} \subset X$ la fibre et on a la spécialisation du groupe de Brauer $i_{t}^{*}: \operatorname{Br}(X) \rightarrow \operatorname{Br}\left(X_{t}\right)$. La proposition suivante dit que, si la fibration $f$ est compatible avec des actions des groupes linéaires, alors les sous-groupes de Brauer invariants sont compatibles avec la spécialisation du groupe de Brauer.

Proposition 4.4. ([C18, Prop. 3.13]) Soit $1 \rightarrow G_{0} \stackrel{\phi}{\rightarrow} G \stackrel{\psi}{\rightarrow} T \rightarrow 1$ une suite exacte de groupes linéaires connexes avec $T$ un tore. Soient $X$ une $G$-variété lisse géométriquement intègre et $X \stackrel{f}{\rightarrow} T$ un $G$-morphisme. Notons $\mathrm{Br}_{1}(G) \stackrel{\phi_{*}}{\rightarrow} \mathrm{Br}_{1}\left(G_{0}\right)$ l'homomorphisme induit par $\phi$. Alors, pour tout $t \in T(k)$, on a

(1) la fibre $i_{t}: X_{t} \subset X$ est $G_{0}$-invariante;

(2) on a une suite exacte naturelle

$$
\mathrm{Br}_{e}(T) \rightarrow \operatorname{Br}_{G}(X) \stackrel{i_{t}^{*}}{\rightarrow} \operatorname{Br}_{G_{0}}\left(X_{t}\right) \rightarrow \operatorname{coker}\left(\phi_{*}\right)
$$

(3) ([C18, Lem. 5.5]) si $k$ est un corps de nombres et $H^{3}\left(k, T^{*}\right)=0$, on a $\operatorname{coker}\left(\phi_{*}\right)=0$ et $i_{t}^{*}$ est surjectif.

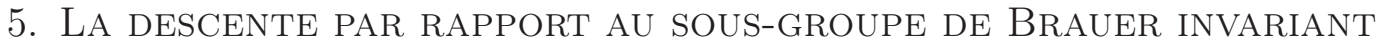

Dans toute cette section, $k$ est un corps de nombres. Sauf mention explicite du contraire, une variété est une $k$-variété.

La méthode de descente des points adéliques est établie par Colliot-Thélène et Sansuc dans [CTSb]. Dans [C18], l'auteur étudie la méthode de descente des points adéliques orthogonaux aux sous-groupes de Brauer invariants et établit le résultat : pour un groupe linéaire connexe $G$, une variété lisse géométriquement intègre $Z$ et un $G$-torseur $p: X \rightarrow Z$,

(1) on a $([\mathrm{C} 18$, Thm. 5.9]) :

$$
Z\left(\mathbf{A}_{k}\right)^{\operatorname{Br}(Z)}=\cup_{\sigma \in H^{1}(k, G)} p_{\sigma}\left(X_{\sigma}\left(\mathbf{A}_{k}\right)^{\operatorname{Br}_{G_{\sigma}}\left(X_{\sigma}\right)}\right) ;
$$

(2) si $G$ est un tore quasi-trivial, on a ([C18, Prop. 5.2])

$$
Z\left(\mathbf{A}_{k}\right)^{\left(p^{*}\right)^{-1} B}=p\left(X\left(\mathbf{A}_{k}\right)^{B}\right)
$$

pour tout sous-groupe $B \subset \operatorname{Br}_{G}(X)$, où $p^{*}: \operatorname{Br}(Z) \rightarrow \operatorname{Br}(X)$;

(3) pour tout homomorphisme surjectif $\psi: H \rightarrow G$ de groupes linéaires connexes, on a ([CLX, Thm. 5.1]) :

$$
G\left(\mathbf{A}_{k}\right)^{\operatorname{Br}_{1}(G)}=\psi\left(H\left(\mathbf{A}_{k}\right)^{\operatorname{Br}_{1}(H)}\right) \cdot G(k) .
$$

La proposition 5.1 et la proposition 5.5 suivantes sont quelques variantes de ce résultat. Plus précisément, la proposition 5.1 est une variante de (2) pour $G$ un groupe fini commutatif et sa démonstration utilise (2) et (3) mais pas (1). La proposition 5.5 est une variante de (1) en 
remplaçant "Br" par "ét, Br" et en remplaçant " $\mathrm{Br}_{G}$ " par " $G$-ét, $\mathrm{Br}_{G}$ ", donc elle est une version limite de (1) pour tout $k$-torseur $Z^{\prime} \rightarrow Z$ sous un $k$-groupe fini, et sa démonstration utilise $(1)$ mais pas (2) et (3).

Proposition 5.1. Soient $G, H$ deux groupes linéaires connexes et $\psi: H \rightarrow G$ un homomorphisme surjectif de noyau $S$ fini. Soient $X$ (resp. $Y$ ) une $G$-variété (resp. H-variété) lisse géométriquement intègre et $f: Y \rightarrow X$ un $H$-morphisme tels que $Y$ soit un $S$-torseur sur $X$, où l'action de $S$ est induite par l'action de $H$. Alors, pour tout $\sigma \in H^{1}(k, S)$, le tordu $Y_{\sigma}$ est une $H$-variété et on a:

$$
X\left(\mathbf{A}_{k}\right)^{\mathrm{Br}_{G}(X)}=\cup_{\sigma \in H^{1}(k, S)} f_{\sigma}\left(Y_{\sigma}\left(\mathbf{A}_{k}\right)^{\mathrm{Br}_{H}\left(Y_{\sigma}\right)}\right) .
$$

Démonstration. On construira les diagrammes ci-dessous
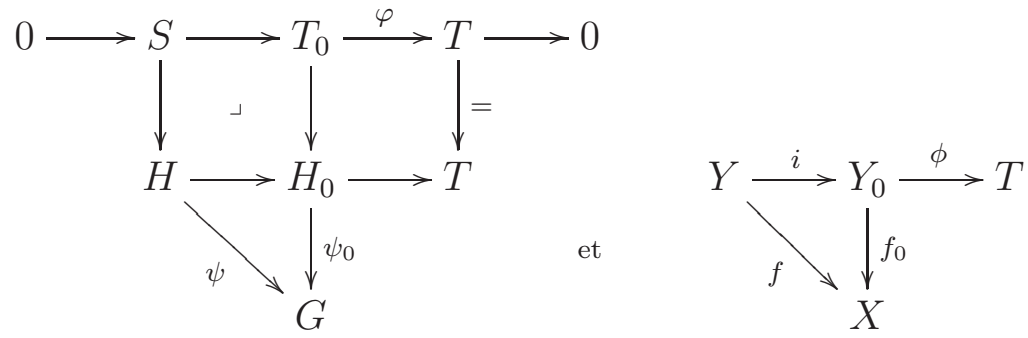

où le diagramme à gauche est un diagramme de groupes algébriques, le diagramme à droite est un diagramme de variétés lisses, chaque groupe dans le diagramme à gauche agit sur la variété dans le diagramme à droite avec le même position et ces actions sont compatibles avec tous les morphismes.

Puisque $H$ est connexe, $S$ est contenu dans le centre de $H$. Donc $S$ est commutatif. Une résolution coflasque ([CTSa, Prop. 1.3]) induit une suite exacte

$$
0 \rightarrow S \rightarrow T_{0} \stackrel{\varphi}{\rightarrow} T \rightarrow 0
$$

où $T_{0}$ est un tore quasi-trivial et $T$ est un tore coflasque, i.e. $H^{1}\left(k^{\prime}, T^{*}\right)=0$ pour toute extension $k^{\prime} / k$. Puisque $H^{3}\left(k, T^{*}\right) \cong \prod_{v \in \infty_{k}} H^{3}\left(k_{v}, T^{*}\right) \cong \prod_{v \in \infty_{k}} H^{1}\left(k_{v}, T^{*}\right)$ ([C18, Lem. 5.4]), on a $H^{3}\left(k, T^{*}\right)=0$.

Soit $H_{0}:=H \times{ }^{S} T_{0}$. Alors $H_{0}$ est un groupe linéaire connexe et $H \stackrel{\psi}{\rightarrow} G$ induit une suite exacte

$$
1 \rightarrow T_{0} \rightarrow H_{0} \stackrel{\psi_{0}}{\rightarrow} G \rightarrow 1
$$

Soit $Y_{0}:=Y \times^{S} T_{0}$. Notons $i: Y \rightarrow Y_{0}$ l'immersion fermée canonique. Alors $Y_{0}$ est une $H_{0^{-}}$ variété et $f$ induit un $H_{0}$-morphisme $Y_{0} \stackrel{f_{0}}{\longrightarrow} X$ tels que $f_{0}$ est un $T_{0}$-torseur. D'après (5.2) et la proposition 4.3 , on a

$$
X\left(\mathbf{A}_{k}\right)^{\mathrm{Br}_{G}(X)}=f_{0}\left(Y_{0}\left(\mathbf{A}_{k}\right)^{\mathrm{Br}_{H_{0}}\left(Y_{0}\right)}\right) .
$$

L'isomorphisme $Y_{0} \times{ }^{T_{0}} T \cong Y \times{ }^{S} T_{0} \times{ }^{T_{0}} T \cong X \times T$ induit un $T_{0}$-morphisme $\phi: Y_{0} \rightarrow T$ tel que $\phi^{-1}\left(e_{T}\right)=i(Y)$. D'après des arguments classiques (voir la démonstration de [C18, Thm. $5.9])$, pour tout $t \in T(k)$, on a $\phi^{-1}(t) \cong Y_{\partial(t)}$ et le morphisme $\phi^{-1}(t) \hookrightarrow Y_{0} \stackrel{f_{0}}{\rightarrow} X$ est exactement $f_{\partial(t)}$, où $\partial: T(k) \rightarrow H^{1}(k, S)$ est l'homomorphisme induit par (5.4). Puisque $H^{3}\left(k, T^{*}\right)=0$, d'après la proposition 4.4, $\phi^{-1}(t)$ est une $H$-variété et l'homomorphisme canonique $\operatorname{Br}_{H_{0}}\left(Y_{0}\right) \rightarrow$ $\operatorname{Br}_{H}\left(\phi^{-1}(t)\right)$ est surjectif pour tout $t \in T(k)$. 


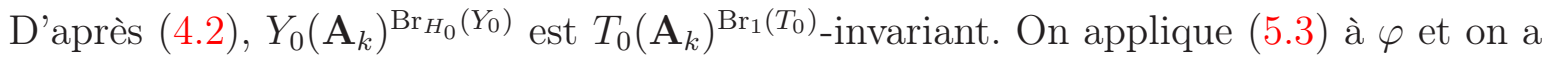

$$
T\left(\mathbf{A}_{k}\right)^{\operatorname{Br}_{1}(T)}=\varphi\left(T_{0}\left(\mathbf{A}_{k}\right)^{\operatorname{Br}_{1}\left(T_{0}\right)}\right) \cdot T(k) .
$$

Puisque $\phi\left(Y_{0}\left(\mathbf{A}_{k}\right)^{\mathrm{Br}_{H_{0}}\left(Y_{0}\right)}\right) \subset T\left(\mathbf{A}_{k}\right)^{\mathrm{Br}_{1}(T)}$, on a :

$$
Y_{0}\left(\mathbf{A}_{k}\right)^{\mathrm{Br}_{H_{0}}\left(Y_{0}\right)}=T_{0}\left(\mathbf{A}_{k}\right)^{\operatorname{Br}_{1}\left(T_{0}\right)} \cdot\left(\sqcup_{t \in T(k)} \phi^{-1}(t)\left(\mathbf{A}_{k}\right)^{\mathrm{Br}_{H}\left(\phi^{-1}(t)\right)}\right),
$$

et donc $X\left(\mathbf{A}_{k}\right)^{\operatorname{Br}_{G}(X)}=f_{0}\left[\sqcup_{t \in T(k)} \phi^{-1}(t)\left(\mathbf{A}_{k}\right)^{\operatorname{Br}_{H}\left(\phi^{-1}(t)\right)}\right]=\cup_{t \in T(k)} f_{\partial(t)}\left[Y_{\partial(t)}\left(\mathbf{A}_{k}\right)^{\operatorname{Br}_{H}\left(Y_{\partial(t)}\right)}\right]$.

Rappelons la définition de $X\left(\mathbf{A}_{k}\right)^{G-e ́ t, B r}{ }_{G}$ dans (1.4).

Pour toute variété lisse $X$, définissons $X\left(\mathbf{A}_{k}^{n c}\right)$ l'espace des points adéliques de $X$ hors des places complexes, i.e. on a $X\left(\mathbf{A}_{k}\right) \cong\left(\prod_{v \text { complexe }} X\left(k_{v}\right)\right) \times X\left(\mathbf{A}_{k}^{n c}\right)$. De plus, on a :

$$
X\left(\mathbf{A}_{k}\right)^{o b} \cong\left(\prod_{v \text { complexe }} X\left(k_{v}\right)\right) \times X\left(\mathbf{A}_{k}^{n c}\right)^{o b}
$$

pour l'obstruction $o b=\operatorname{Br}(X)$ ou $o b=\operatorname{Br}_{1}(X)$ ou $o b=$ ét, $\operatorname{Br}$ ou, si $X$ est une $G$-variété pour un groupe linéaire connexe $G$, pour $o b=\operatorname{Br}_{G}(X)$ ou ob $=G$ - ét, $\operatorname{Br}_{G}$.

Le lemme suivant est bien connu (voir [D09b, Lem. 2.2.8] pour une variante).

Lemme 5.2. Soient $X$ une variété lisse et $\left\{X_{i}\right\}_{i \in I}$ les composantes connexes de $X$ telles que $X_{i}$ soit géométriquement intègre pour tout $i \in I$. Alors on $a$ :

$$
X\left(\mathbf{A}_{k}^{n c}\right)^{\operatorname{Br}_{1}(X)}=\coprod_{i \in I} X_{i}\left(\mathbf{A}_{k}^{n c}\right)^{\operatorname{Br}_{1}\left(X_{i}\right)}, \quad X\left(\mathbf{A}_{k}^{n c}\right)^{\text {ét,Br }}=\coprod_{i \in I} X_{i}\left(\mathbf{A}_{k}^{n c}\right)^{\text {ét,Br }}
$$

et, si $X$ est une $G$-variété pour un groupe linéaire connexe $G$, on a :

$$
X\left(\mathbf{A}_{k}^{n c}\right)^{\operatorname{Br}_{G}(X)}=\coprod_{i \in I} X_{i}\left(\mathbf{A}_{k}^{n c}\right)^{\operatorname{Br}_{G}\left(X_{i}\right)} \quad \text { et } \quad X\left(\mathbf{A}_{k}^{n c}\right)^{G-\text { ét,Br}}=\coprod_{i \in I} X_{i}\left(\mathbf{A}_{k}^{n c}\right)^{G-e ́ t, B_{G}} .
$$

Démonstration. Puisque le groupe de Brauer (resp. le sous-groupe de Brauer G-invariant, resp. l'ensemble des $F$-torseurs, resp. l'ensemble des $F$-torseurs $G$-compatibles pour un $k$-groupe fini $F$ ) de $X$ est la somme directe de celui des composantes connexes de $X$, on obtient l'inclusion $\supset$ dans les quatre cas ci-dessus.

Par ailleurs, soit $\pi_{0}(X)$ le schéma des composantes connexes géométriques de $X$, i.e. $\pi_{0}(X)$ est un $k$-schéma fini étale et il existe un $k$-morphisme surjectif $\phi: X \rightarrow \pi_{0}(X)$ de fibres géométriquement intègres. Pour tout $k$-schéma $V$ fini étale connexe, $V\left(\mathbf{A}_{k}^{n c}\right) \neq \emptyset$ implique $V \cong S p e c k$. D'après [LX, Prop. 3.3] (un résultat inspiré par Stoll), on a $\pi_{0}(X)\left(\mathbf{A}_{k}^{n c}\right)^{\operatorname{Br}\left(\pi_{0}(X)\right)}=\pi_{0}(X)(k)$. Par définition, $\phi^{*}\left(\operatorname{Br}\left(\pi_{0}(X)\right)\right) \subset \operatorname{Br}_{1}(X), \phi^{*}\left(\operatorname{Br}\left(\pi_{0}(X)\right)\right) \subset \operatorname{Br}_{G}(X)$ et donc on obtient l'inclusion $\subset$.

Les deux lemmes suivants sont bien connus.

Lemme 5.3. Soient $X$ une variété lisse, $L$ un groupe linéaire quelconque et $h: V \rightarrow X$ un $L$-torseur. Alors, pour tout $x \in X\left(\mathbf{A}_{k}\right)$, l'ensemble $\left\{\sigma \in H^{1}(k, L): x \in h_{\sigma}\left(V_{\sigma}\left(\mathbf{A}_{k}\right)\right)\right\}$ est fini.

Démonstration. Le résultat découle du fait que, pour tout $\sigma \in H^{1}(k, L)$, l'ensemble $\amalg^{1}\left(k, L_{\sigma}\right)$ est fini ([Se65, §III.4.6]).

Voyons [Sk01, Prop. 5.3.2] et [CDX, Lem. 6.3] pour des résultats similaires. 
Lemme 5.4. (M. Stoll [St], cf. [CDX, Lem. 7.1]) Soit $X$ une variété lisse géométriquement intègre, $F$ un k-groupe fini et $f: Y \rightarrow X$ un $F$-torseur. Supposons qu'il existe un $x \in X\left(\mathbf{A}_{k}\right)^{{ }^{e} t, B r}$. Alors il existe un $\sigma \in H^{1}(k, F)$, un sous-groupe fermé $F^{\prime} \subset F_{\sigma}$, une composante connexe $Y^{\prime} \subset Y_{\sigma}$ tels que $Y^{\prime}$ soit géométriquement intègre et $F^{\prime}$-invariant, $f^{\prime}:=\left.f_{\sigma}\right|_{Y^{\prime}}: Y^{\prime} \rightarrow X$ soit un $F^{\prime}$-torseur et que $x \in f^{\prime}\left(Y^{\prime}\left(\mathbf{A}_{k}\right)^{\operatorname{Br}\left(Y^{\prime}\right)}\right)$.

La proposition suivante est une étape intermédiaire importante dans la démonstration du théorème 1.1.

Proposition 5.5. Soient $G$ un groupe linéaire connexe, $Z$ une variété lisse géométriquement intègre et $p: X \rightarrow Z$ un $G$-torseur. Alors :

$$
Z\left(\mathbf{A}_{k}\right)^{\text {ét,Br }}=\cup_{\sigma \in H^{1}(k, G)} p_{\sigma}\left(X_{\sigma}\left(\mathbf{A}_{k}\right)^{G_{\sigma}-\text { ét, } \operatorname{Br}_{G_{\sigma}}}\right) .
$$

Démonstration. L'inclusion $\supset$ découle du fait que, pour tout torseur $V \rightarrow Z$ sous un $k$-groupe fini, l'image réciproque $X \times_{Z} V \rightarrow X$ est $G$-compatible.

Pour l'inclusion $\subset$, on peut supposer que $Z\left(\mathbf{A}_{k}\right)^{\text {ét,Br }} \neq \emptyset$.

On fixe un point $z \in Z\left(\mathbf{A}_{k}\right)^{\text {ét,Br }}$.

Soit $\Delta$ l'ensemble des $\sigma \in H^{1}(k, G)$ tels que $p_{\sigma}^{-1}(z) \cap X_{\sigma}\left(\mathbf{A}_{k}\right)^{\operatorname{Br}_{G_{\sigma}}\left(X_{\sigma}\right)} \neq \emptyset$. Alors $\Delta \neq \emptyset$ par (5.1). Pour tout $\sigma \in \Delta$, on fixe un point $x_{\sigma} \in p_{\sigma}^{-1}(z) \cap X_{\sigma}\left(\mathbf{A}_{k}\right)^{\operatorname{Br}_{G_{\sigma}}\left(X_{\sigma}\right)}$. Ceci induit un isomorphisme :

$$
\Psi_{\sigma}: G\left(\mathbf{A}_{k}\right) \rightarrow p_{\sigma}^{-1}(z)\left(\mathbf{A}_{k}\right): g \mapsto g \cdot x_{\sigma}
$$

Notons :

$$
E_{0, \sigma}:=\Psi_{\sigma}^{-1}\left(p_{\sigma}^{-1}(z) \cap X_{\sigma}\left(\mathbf{A}_{k}\right)^{\mathrm{Br}_{G_{\sigma}}\left(X_{\sigma}\right)}\right) \quad \text { et } \quad E_{0}:=\sqcup_{\sigma \in \Delta} E_{0, \sigma} .
$$

Pour tout $\sigma \in \Delta$, soit $G_{\sigma}\left(\mathbf{A}_{k}\right) \stackrel{a_{\sigma}}{\longrightarrow} \operatorname{Hom}\left(\operatorname{Br}_{a}\left(G_{\sigma}\right), \mathbb{Q} / \mathbb{Z}\right)$ l'homomorphisme induit par l'accouplement de Brauer-Manin. Donc $\operatorname{Ker}\left(a_{\sigma}\right)=G_{\sigma}\left(\mathbf{A}_{k}\right)^{\operatorname{Br}_{a}\left(G_{\sigma}\right)}$. Notons

$$
K_{a, \Delta}:=\prod_{\sigma \in \Delta} \operatorname{Ker}\left(a_{\sigma}\right) \quad \text { et } \quad G_{\Delta}\left(\mathbf{A}_{k}\right):=\sqcup_{\sigma \in \Delta} G_{\sigma}\left(\mathbf{A}_{k}\right) .
$$

Définissons l'action de $\operatorname{Ker}\left(a_{\sigma}\right)$ sur $G_{\sigma}\left(\mathbf{A}_{k}\right)$ par la multiplication à gauche. Ceci induit une unique action de $K_{a, \Delta}$ sur $G_{\Delta}\left(\mathbf{A}_{k}\right)$ telle que l'action de $\operatorname{Ker}\left(\sigma_{1}\right)$ sur $G_{\sigma_{2}}\left(\mathbf{A}_{k}\right)$ soit l'identité pour tous $\sigma_{1} \neq \sigma_{2}$. D'après (4.2), $E_{0}$ est $K_{a, \Delta}$-invariant.

Soit $\mathcal{S}$ l'ensemble des couples $(F, V \stackrel{f}{\rightarrow} Z$ ) avec $F$ un $k$-groupe fini et $V \stackrel{f}{\rightarrow} Z$ un $F$-torseur tel que $V$ soit géométriquement intègre. On définit un ordre partiel : pour tous $\left(F_{1}, V_{1}\right),\left(F_{2}, V_{2}\right) \in$ $\mathcal{S}$, on a $\left(F_{1}, V_{1}\right) \leq\left(F_{2}, V_{2}\right)$ si et seulement s'il existe un $\sigma \in H^{1}\left(k, F_{1}\right)$ et un homomorphisme surjectif $\phi: F_{2} \rightarrow F_{1, \sigma}$ tels que $\phi_{*}\left(\left[V_{2}\right]\right)=\left[V_{1, \sigma}\right]$.

Pour tout $(\delta, \sigma) \in H^{1}(k, F) \times H^{1}(k, G)$, soit $Y_{\sigma, \delta}:=X_{\sigma} \times_{Z} V_{\delta}$. On a un diagramme commutatif de $F_{\delta} \times G_{\sigma^{-}}$variétés et de $F_{\delta} \times G_{\sigma}$-morphismes :

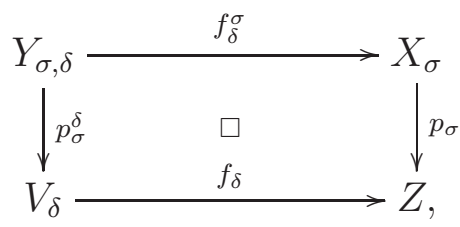

tel que toute verticale soit un $G_{\sigma^{-}}$-torseur et que toute horizontale soit un $F_{\delta^{-}}$-torseur. 
Pour tout $(F, V \stackrel{f}{\rightarrow} Z) \in \mathcal{S}$ et tout $\sigma \in \Delta$, notons

$$
E_{F, V, \sigma}:=\Psi_{\sigma}^{-1}\left(p_{\sigma}^{-1}(z) \cap\left[\cup_{\delta \in H^{1}(k, F)} f_{\delta}^{\sigma}\left(Y_{\sigma, \delta}\left(\mathbf{A}_{k}\right)^{\operatorname{Br}_{G_{\sigma}}\left(Y_{\sigma, \delta}\right)}\right)\right]\right) \subset G_{\sigma}\left(\mathbf{A}_{k}\right)
$$

et $E_{F, V}:=\sqcup_{\sigma \in \Delta} E_{F, V, \sigma} \subset G_{\Delta}\left(\mathbf{A}_{k}\right)$.

Lemme 5.6. Pour tout $(F, V \stackrel{f}{\rightarrow} Z) \in \mathcal{S}$, on a :

(1) l'ensemble $E_{F, V}$ est un sous-ensemble non vide fermé $K_{a, \Delta}$-invariant de $E_{0}$;

(2) pour tout $\left(F_{1}, V_{1}\right) \in \mathcal{S}$ vérifiant $(F, V) \leq\left(F_{1}, V_{1}\right)$, on a $E_{F_{1}, V_{1}} \subset E_{F, V}$;

(3) l'ensemble $\mathcal{S}$ est un ensemble ordonné filtrant.

Démonstration. Pour tout $\delta \in H^{1}(k, F)$ et tout $\sigma \in H^{1}(k, G)$, le morphisme $f_{\delta}^{\sigma}$ est fini. D'après (4.2), $Y_{\sigma, \delta}\left(\mathbf{A}_{k}\right)^{\operatorname{Br}_{G \sigma}\left(Y_{\sigma, \delta}\right)}$ est $\operatorname{Ker}\left(a_{\sigma}\right)$-invariant et

$$
f_{\delta}^{\sigma}\left(Y_{\sigma, \delta}\left(\mathbf{A}_{k}\right)^{\operatorname{Br}_{G_{\sigma}}\left(Y_{\sigma, \delta}\right)}\right) \subset X_{\sigma}\left(\mathbf{A}_{k}\right)^{\operatorname{Br}_{G_{\sigma}}\left(X_{\sigma}\right)}
$$

est fermé ([Cod, Prop. 4.4]) et $\operatorname{Ker}\left(a_{\sigma}\right)$-invariant. Ainsi

$$
\Psi_{\sigma}^{-1}\left[p_{\sigma}^{-1}(z) \cap f_{\delta}^{\sigma}\left(Y_{\sigma, \delta}\left(\mathbf{A}_{k}\right)^{\operatorname{Br}_{G \sigma}\left(Y_{\sigma, \delta}\right)}\right)\right] \subset E_{0, \sigma}
$$

est fermé et $\operatorname{Ker}\left(a_{\sigma}\right)$-invariant.

Appliquant (5.1) et le lemme 5.3 à $Y_{\sigma, \delta} \stackrel{p_{\sigma}^{\delta}}{\longrightarrow} V_{\delta}$, il existe au moins un et au plus un nombre fini de $(\delta, \sigma) \in H^{1}(k, F) \times H^{1}(k, G)$ tels que

$$
\left(f_{\delta} \circ p_{\sigma}^{\delta}\right)^{-1}(z) \cap Y_{\sigma, \delta}\left(\mathbf{A}_{k}\right)^{\operatorname{Br}_{G_{\sigma}}\left(Y_{\sigma, \delta}\right)} \neq \emptyset .
$$

Alors $p_{\sigma}^{-1}(z) \cap X_{\sigma}\left(\mathbf{A}_{k}\right)^{\operatorname{Br}_{G_{\sigma}}\left(X_{\sigma}\right)} \neq \emptyset$ et donc un tel $\sigma$ est dans $\Delta$. Alors $E_{F, V} \neq \emptyset$ et (1) découle du premier paragraphe.

L'énoncé (2) découle de la fonctorialité de l'accouplement de Brauer-Manin.

Pour tous $\left(F_{1}, V_{1}\right),\left(F_{2}, V_{2}\right) \in \mathcal{S}$, on a un $F_{1} \times F_{2}$-torseur $V_{1} \times_{Z} V_{2} \rightarrow Z$. Par hypothèse, il existe un $\left(\sigma_{1}, \sigma_{2}\right) \in H^{1}\left(k, F_{1}\right) \times H^{1}\left(k, F_{2}\right)$ tel que $\left(V_{1, \sigma_{1}} \times{ }_{Z} V_{2, \sigma_{2}}\right)\left(\mathbf{A}_{k}\right)^{\operatorname{Br}\left(V_{1, \sigma_{1}} \times{ }_{Z} V_{2, \sigma_{2}}\right)} \neq \emptyset$. D'après le lemme 5.2 et (5.5), il existe un $k$-sous-groupe fermé $F_{3} \subset F_{1, \sigma_{1}} \times F_{2, \sigma_{2}}$ et une composante connexe $V_{3} \subset V_{1, \sigma_{1}} \times_{Z} V_{2, \sigma_{2}}$ tels que $V_{3}$ soit géométriquement intègre et que $V_{3} \rightarrow Z$ soit un $F_{3}$-torseur compatible avec l'action de $F_{1, \sigma_{1}} \times F_{2, \sigma_{2}}$ sur $V_{1, \sigma_{1}} \times_{Z} V_{2, \sigma_{2}}$. Alors le morphisme $h_{1}: V_{3} \subset V_{1, \sigma_{1}} \times_{Z} V_{2, \sigma_{2}} \rightarrow V_{1, \sigma_{1}}$ est compatible avec $\phi_{1}: F_{3} \subset F_{1, \sigma_{1}} \times F_{2, \sigma_{2}} \rightarrow F_{1, \sigma_{1}}$. Puisque $V_{1, \sigma_{1}}$ est géométriquement intègre, le morphisme $h_{1}$ est surjectif et donc $\phi_{1}$ est surjectif. Alors $\left[V_{1, \sigma_{1}}\right]=\phi_{1, *}\left(\left[V_{3}\right]\right)$ et $\left(F_{1}, V_{1}\right) \leq\left(F_{3}, V_{3}\right)$. Par ailleurs, $\left(F_{2}, V_{2}\right) \leq\left(F_{3}, V_{3}\right)$, d'où l'énoncé $(3)$.

Soient $\mathcal{B}:=\sqcup_{\sigma \in \Delta} \operatorname{Hom}\left(\operatorname{Br}_{a}\left(G_{\sigma}\right), \mathbb{Q} / \mathbb{Z}\right)$ et

$$
a_{\Delta}: G_{\Delta}=\sqcup_{\sigma \in \Delta} G_{\sigma}\left(\mathbf{A}_{k}\right) \stackrel{\sqcup_{\sigma \in \Delta} a_{\sigma}}{\longrightarrow} \sqcup_{\sigma \in \Delta} \operatorname{Hom}\left(\operatorname{Br}_{a}\left(G_{\sigma}\right), \mathbb{Q} / \mathbb{Z}\right)=\mathcal{B} .
$$

En tant qu'ensembles, on a $\operatorname{Im}\left(a_{\Delta}\right) \cong K_{a, \Delta} \backslash G_{\Delta}$. L'espace $\operatorname{Hom}\left(\operatorname{Br}_{a}\left(G_{\sigma}\right), \mathbb{Q} / \mathbb{Z}\right)$ est compact, car $\operatorname{Br}_{a}\left(G_{\sigma}\right)$ est discret. D'après le lemme 5.3, $\Delta$ est fini et donc $\mathcal{B}$ est compact. Puisque $a_{\sigma}$ est continu et ouvert ([C18, Lem. 4.1]), l'application $a_{\Delta}$ est ouverte. Donc l'image d'un sousensemble fermé $K_{a, \Delta}$-invariant est fermée. Alors $a_{\Delta}\left(E_{F, V}\right) \subset \mathcal{B}$ est fermé non vide pour tout $(F, V) \in \mathcal{S}$. Puisque $\mathcal{B}$ est compact et que $\mathcal{S}$ est un ensemble ordonné filtrant, d'après le lemme 5.6 (2), l'intersection

$$
\bigcap_{(F, V) \in \mathcal{S}} a_{\Delta}\left(E_{F, V}\right) \neq \emptyset \quad \text { et donc } \quad E_{\infty}:=\bigcap_{(F, V) \in \mathcal{S}} E_{F, V} \neq \emptyset .
$$


Il existe un $\sigma \in \Delta$ tel que $E_{\infty} \cap E_{\sigma} \neq \emptyset$.

Soient $g \in E_{\infty} \cap E_{\sigma}$ et $x:=\Psi_{\sigma}(g)=g \cdot x_{\sigma}$. Alors $p_{\sigma}(x)=z$ et, d'après (5.6), on a

$$
x \in \bigcap_{(F, V) \in \mathcal{S}}\left[\bigcup_{\delta \in H^{1}(k, F)} f_{\delta}^{\sigma}\left(Y_{\sigma, \delta}\left(\mathbf{A}_{k}\right)^{\operatorname{Br}_{G_{\sigma}}\left(Y_{\sigma, \delta}\right)}\right)\right] .
$$

D'après le corollaire 3.7, tout torseur $G$-compatible sous un $k$-groupe fini sur $X$ provient d'un torseur sur Z. D'après le lemme de Stoll (Lem. 5.4), il suffit de considérer les torseurs géométriquement intègres. Donc $x \in X_{\sigma}\left(\mathbf{A}_{k}\right)^{G_{\sigma}-\text { ét, } \mathrm{Br}_{G_{\sigma}}}$, d'où le résultat.

La proposition suivante est une généralisation de [CDX, Rem. 7.5].

Proposition 5.7. Soit $X$ une variété lisse géométriquement intègre. Soit

$$
1 \rightarrow N \rightarrow L \stackrel{\psi}{\rightarrow} F \rightarrow 1
$$

une suite exacte de groupes linéaires avec F fini. Soient $V \rightarrow X$ un L-torseur et $Y:=V / N \rightarrow X$ le F-torseur induit par $\psi$, i.e. $[Y]=\psi_{*}([V])$. Faisons l'une ou l'autre des hypothèses :

(1) le groupe $N$ est connexe;

(2) le groupe $L$ est fini et $N$ est contenu dans le centre de $L$.

Alors, pour tout $\sigma \in H^{1}(k, F)$ avec $Y_{\sigma}\left(\mathbf{A}_{k}\right)^{\operatorname{Br}_{1}\left(Y_{\sigma}\right)} \neq \emptyset$, il existe un $\alpha \in H^{1}(k, L)$ tel que $\psi_{*}(\alpha)=\sigma$.

Démonstration. Le cas où $N$ est connexe est exactement [CDX, Rem. 7.5].

On considère le cas (2). Dans ce cas, $N$ est un $k$-groupe fini commutatif. La résolution flasque ([CTSa, Prop. 1.3]) donne une suite exacte $0 \rightarrow N \rightarrow T \rightarrow T_{0} \rightarrow 0$ avec $T$ un tore et $T_{0}$ un tore quasi-trivial. Soit $L^{\prime}:=L \times{ }^{N} T$. Alors $L^{\prime}$ est un groupe linéaire, car $N$ est contenu dans le centre de $L$. Ceci induit un diagramme commutatif de suites exactes et de colonnes exactes :

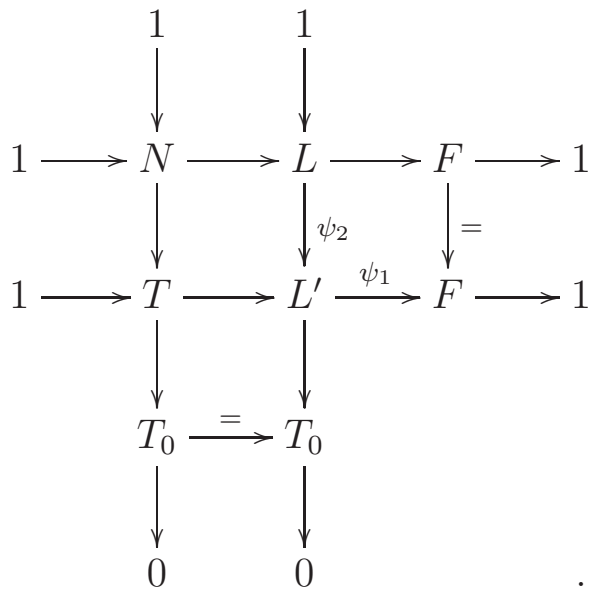

Appliquons le cas (1) au $L^{\prime}$-torseur $\psi_{2, *}([V])$. On obtient un $\beta \in H^{1}\left(k, L^{\prime}\right)$ tel que $\psi_{1, *}(\beta)=\sigma$. Puisque $H^{1}\left(k, T_{0}\right)=0$, il existe un $\alpha \in H^{1}(k, L)$ tel que $\psi_{2, *}(\alpha)=\beta$ et donc $\psi_{*}(\alpha)=\sigma$.

La proposition 5.7 (2) et la formule (4.1) impliquent directement :

Corollaire 5.8. Sous les hypothèses de la proposition 5.7 (2), soit $G$ un groupe linéaire. Pour tout $\sigma \in H^{1}(k, F)$, s'il existe une action de $G$ sur $Y_{\sigma}$ telle que $Y_{\sigma}\left(\mathbf{A}_{k}\right)^{\operatorname{Br}_{G}\left(Y_{\sigma}\right)} \neq \emptyset$, alors il existe un $\alpha \in H^{1}(k, L)$ tel que $\psi_{*}(\alpha)=\sigma$. 


\section{DÉmonstration DU ThÉORÈme 1.4}

Dans toute cette section, $k$ est un corps de nombres. Sauf mention explicite du contraire, une variété est une $k$-variété.

Dans toute cette section, $G$ est un $k$-groupe linéaire connexe et $(X, \rho)$ une $G$-variété lisse géométriquement intègre.

Pour tout $k$-groupe fini $F$ et tout $F$-torseur $f: Y \rightarrow X$, soit $\left(H_{Y} \stackrel{\psi_{Y}}{\longrightarrow} G\right)$ le groupe minimal compatible au $F$-torseur $Y$ (cf. Définition 3.9). Pour tout $\sigma \in H^{1}(k, F)$, le $F_{\sigma}$-torseur $f_{\sigma}$ : $Y_{\sigma} \rightarrow X$ est $H_{Y}$-compatible, i.e. il existe une unique action de $H_{Y}$ sur $Y_{\sigma}$ telle que $f_{\sigma}$ soit un $H_{Y}$-morphisme.

Dans $\S 1$, on a défini $X\left(\mathbf{A}_{k}\right)^{\text {ét,Br }}\left(\right.$ cf. (1.1)) et $X\left(\mathbf{A}_{k}\right)^{G \text {-ét,Br}} \operatorname{Br}_{G}$ (cf. (1.4)). On définit

$$
X\left(\mathbf{A}_{k}\right)^{\text {ét, Br }_{G}}:=\bigcap_{\substack{f: Y \\ F \text { fini }}} \bigcup_{\vec{F}_{X},} \bigcup_{\sigma \in H^{1}(k, F)} f_{\sigma}\left(Y_{\sigma}\left(\mathbf{A}_{k}\right)^{\operatorname{Br}_{H_{Y}}\left(Y_{\sigma}\right)}\right)
$$

et

$$
X\left(\mathbf{A}_{k}\right)^{\text {c.c.,ét, } \mathrm{Br}_{G}}:=\bigcap_{\substack{f: Y, \underline{F}_{X} \\ F \text { fini commutatif, } Y \text { géo. connexe }}} \bigcup_{\sigma \in H^{1}(k, F)} f_{\sigma}\left(Y_{\sigma}\left(\mathbf{A}_{k}\right)^{\mathrm{Br}_{H_{Y}}\left(Y_{\sigma}\right)}\right)
$$

où geo. connexe signifie géométriquement connexe et c.c. est une abréviation de commutatif connexe. On a directement :

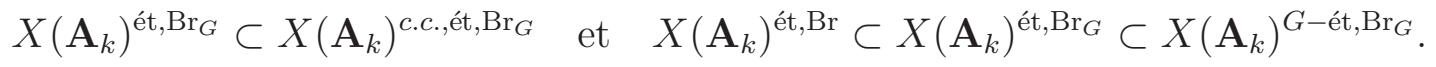

Proposition 6.1. On a $X\left(\mathbf{A}_{k}\right)^{\text {c.c. ét, } \operatorname{Br}_{G}} \subset X\left(\mathbf{A}_{k}\right)^{\operatorname{Br}(X)}$.

Démonstration. Il suffit de montrer que, pour tout $\alpha \in \operatorname{Br}(X)$ et tout $x \in X\left(\mathbf{A}_{k}\right)^{\text {c.c.,ét,Br}}{ }_{G}$, on a $\alpha(x)=0$. On fixe un tel $x$ et un tel $\alpha$.

Il existe un entier $n$ tel que $n \cdot \alpha=0$. D'après le corollaire 4.2, il existe un torseur universel de $n$-torsion $\mathcal{T}_{X} \stackrel{f}{\rightarrow} X$ (un $S_{X}$-torseur). Soit $H$ le groupe minimal compatible au $S_{X}$-torseur $\mathcal{T}_{X}$. Par hypothèse, il existe un $\sigma \in H^{1}\left(k, S_{X}\right)$ et un point adélique $t \in \mathcal{T}_{X, \sigma}\left(\mathbf{A}_{k}\right)^{\mathrm{Br}_{H}\left(\mathcal{T}_{X, \sigma}\right)}$ tels que $f_{\sigma}(t)=x$. D'après la proposition 3.12, $f_{\sigma}^{*}(\alpha) \in \operatorname{Br}_{H}\left(\mathcal{T}_{X, \sigma}\right)$. Alors $\alpha(x)=f_{\sigma}^{*}(\alpha)(t)=0$.

Le lemme suivant généralise un résultat de Skorobogatov ([Sk09, Thm. 1.1]) et il généralise aussi [CDX, Prop. 6.6]. Sa démonstration suit l'idée de [Sk09, p. 506] et de [St, Prop. 5.17].

Lemme 6.2. Soient $F$ un k-groupe fini, $f: Y \rightarrow X$ un $F$ torseur et $\left(H_{Y} \stackrel{\psi_{Y}}{\longrightarrow} G\right)$ le groupe minimal compatible au F-torseur $Y$. Supposons que $Y$ est géométriquement intègre. Alors

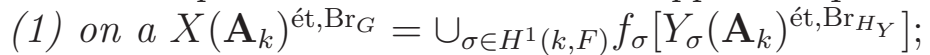

(2) on a $X\left(\mathbf{A}_{k}\right)^{\text {ét,Br }}=\cup_{\sigma \in H^{1}(k, F)} f_{\sigma}\left[Y_{\sigma}\left(\mathbf{A}_{k}\right)^{\text {ét,Br }}\right]$;

(3) si $\psi_{Y}: H_{Y} \stackrel{\sim}{\rightarrow} G$ est un isomorphisme, on a

$$
X\left(\mathbf{A}_{k}\right)^{G-\text { ét, } \mathrm{Br}_{G}}=\cup_{\sigma \in H^{1}(k, F)} f_{\sigma}\left[Y_{\sigma}\left(\mathbf{A}_{k}\right)^{G-\text { ét, } \mathrm{Br}_{G}}\right] .
$$

Démonstration. L'inclusion $\supset$ dans les trois cas est définie par le pullback des torseurs et la fonctorialité de l'accouplement de Brauer-Manin. On considère l'inclusion $\subset$.

Dans le cas (1), il suffit de montrer que, pour tout $x \in X\left(\mathbf{A}_{k}\right)^{\text {ét,Br}}{ }^{G}$, il existe un $\sigma \in H^{1}(k, F)$ et un $y \in Y_{\sigma}\left(\mathbf{A}_{k}\right)^{\text {ét, } \mathrm{Br}_{H_{Y}}}$ tels que $f_{\sigma}(y)=x$. On fixe un tel $x$. 
Pour tout $\sigma \in H^{1}(k, F)$, soient

$$
\Delta_{\sigma}:=f_{\sigma}^{-1}(x) \cap Y_{\sigma}\left(\mathbf{A}_{k}\right), \quad \Sigma:=\left\{\sigma \in H^{1}(k, F): \Delta_{\sigma} \neq \emptyset\right\} \quad \text { et } \quad \Delta:=\sqcup_{\sigma \in \Sigma} \Delta_{\sigma} .
$$

D'après le lemme $5.3, \Delta$ et $\Sigma$ sont finis.

Soit $\mathcal{S}$ l'ensemble des $X$-torseurs sur $Y$ sous $k$-groupes finis i.e. l'ensemble des quintuples $\left(\sigma, E, E \stackrel{\psi}{\rightarrow} F_{\sigma}, V \stackrel{h_{V}}{\rightarrow} X, V \stackrel{h}{\rightarrow} Y_{\sigma}\right)$ avec $\sigma \in H^{1}(k, F), E$ un $k$-groupe fini, $\psi$ un homomorphisme surjectif, $V \stackrel{h_{V}}{\longrightarrow} X$ un $E$-torseur et $h$ un $E$-morphisme sur $X$. Alors $\psi_{*}([V])=\left[Y_{\sigma}\right] \in H^{1}(k, F)$ et $h: V \rightarrow Y_{\sigma}$ est un $\operatorname{Ker}(\psi)$-torseur. Donc $h_{\alpha}: V_{\alpha} \rightarrow Y_{\sigma+\psi_{*}(\alpha)}$ est un $\operatorname{Ker}\left(\psi_{\alpha}\right)$-torseur pour tout $\alpha \in H^{1}(k, E)$. Soit

$$
\Delta_{V}:=\left\{y \in \Delta: \exists \alpha \in H^{1}(k, E) \text { tel que } y \in h_{\alpha}\left(V_{\alpha}\left(\mathbf{A}_{k}\right)^{\mathrm{Br}_{H_{V}}\left(V_{\alpha}\right)}\right)\right\} .
$$

Par l'hypothèse sur $x$, l'ensemble $\Delta_{V}$ est non vide.

On définit un ordre partiel de $\mathcal{S}$ : pour tous $\left(\sigma_{1}, E_{1}, \psi_{1}, V_{1}, h_{1}\right),\left(\sigma_{2}, E_{2}, \psi_{2}, V_{2}, h_{2}\right) \in \mathcal{S}$, on a $\left(\sigma_{1}, E_{1}, \psi_{1}, V_{1}, h_{1}\right) \leq\left(\sigma_{2}, E_{2}, \psi_{2}, V_{2}, h_{2}\right)$ si et seulement si $\sigma_{1}=\sigma_{2}$ et s'il existe un $\alpha \in H^{1}\left(k, E_{1}\right)$, un homomorphisme surjectif $\phi: E_{2} \rightarrow E_{1, \alpha}$ et un $E_{2}$-morphisme $h_{\phi}: V_{2} \rightarrow V_{1, \alpha}$ sur $Y_{\sigma_{1}}$. Dans ce cas, on a $\Delta_{V_{2}} \subset \Delta_{V_{1}}$.

Puisque $\Delta$ est fini, il existe un quintuple $\left(\sigma, E_{0}, \psi_{0}, V_{0}, h_{0}\right)$ dans $S$ tel que $\Delta_{V_{0}}$ soit minimal. On fixe un $y \in \Delta_{V_{0}}$. Après avoir remplacé $\sigma$ par $\sigma+\psi_{0, *}(\alpha)$ pour certain $\alpha \in H^{1}\left(k, E_{0}\right)$, on peut supposer que $y \in Y_{\sigma}\left(\mathbf{A}_{k}\right)$.

Pour tout torseur $Z \stackrel{f_{1}}{\rightarrow} Y_{\sigma}$ sous un $k$-groupe fini $F_{1}$, d'après [Sk09, Prop. 2.3 et (4)], il existe un $\left(\sigma, E, \psi, h_{V}: V \rightarrow X, h\right) \in \mathcal{S}$, un homomorphisme surjectif $\operatorname{Ker}(\psi) \rightarrow F_{1}$ et un $\operatorname{Ker}(\psi)$-morphisme $V \rightarrow Z$ sur $Y_{\sigma}$ avec

$$
V:=R_{Y_{\sigma} \times_{k} F_{\sigma} / Y_{\sigma}}\left(Z \times_{k} F_{\sigma}\right) \cong R_{Y_{\sigma} / X}(Z) \times_{X} Y_{\sigma} \stackrel{h_{V}}{\longrightarrow} X
$$

Ceci induit

$$
\Delta_{V} \subset \cup_{\alpha \in H^{1}(k, \operatorname{Ker}(\psi))} h_{\alpha}\left(V_{\alpha}\left(\mathbf{A}_{k}\right)^{\mathrm{Br}_{H_{V}}\left(V_{\alpha}\right)}\right) \subset \cup_{\alpha \in H^{1}\left(k, F_{1}\right)} f_{1, \alpha}\left(Z_{\alpha}\left(\mathbf{A}_{k}\right)^{\mathrm{Br}_{H_{Z}}\left(Z_{\alpha}\right)}\right) .
$$

Par ailleurs, on a :

$$
\left(\sigma, E_{0}, \psi_{0}, V_{0}, h_{0}\right),(\sigma, E, \psi, V, h) \leq\left(\sigma, E_{0} \times_{F_{\sigma}} E, \psi_{0} \circ\left(i d_{E_{0}} \times_{F_{\sigma}} \psi\right), V_{0} \times_{Y_{\sigma}} V, h_{0} \circ\left(i d_{V_{0}} \times_{Y_{\sigma}} h\right)\right)
$$

dans $\mathcal{S}$, et donc $\Delta_{V_{0}} \supset \Delta_{V_{0} \times_{Y_{\sigma}} V} \subset \Delta_{V}$. Puisque $\Delta_{V_{0}}$ est minimal, on a $\Delta_{V_{0}}=\Delta_{V_{0} \times_{Y_{\sigma}} V} \subset \Delta_{V}$.

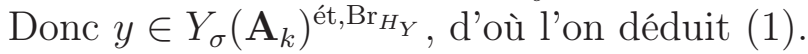

L'énoncé (2) découle du même argument que l'énoncé (1).

Pour (3), d'après le corollaire 3.6, le torseur $V \rightarrow X$ dans (6.1) est G-compatible. L'énoncé (3) découle du même argument que l'énoncé (1).

La proposition suivante généralise un lemme de Stoll ([St], cf. Lem. 5.4).

Proposition 6.3. Soient $G$ un k-groupe linéaire connexe et $(X, \rho)$ une $G$-variété lisse géomé-

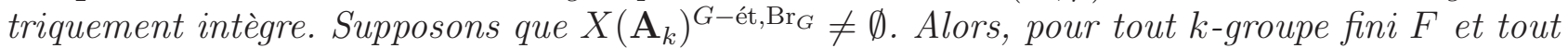
$F$-torseur $Y \rightarrow X$, il existe un $\sigma \in H^{1}(k, F)$ tel qu'il existe une composante connexe $Y^{\prime} \subset Y_{\sigma}$ qui est géométriquement intègre.

De plus, dans ce cas, il existe un sous k-groupe fermé $F^{\prime} \subset F_{\sigma}$ tel que $Y^{\prime}$ soit un $F^{\prime}$-torseur sur $X$, où l'action de $F^{\prime}$ sur $Y^{\prime}$ est induite par l'action de $F_{\sigma}$ sur $Y_{\sigma}$. 
Démonstration. Le morphisme $G \times X \stackrel{\rho}{\rightarrow} X$ induit un homomorphisme $\rho_{\pi_{1}}: \pi_{1}\left(G_{\bar{k}}\right) \rightarrow \pi_{1}(X)$. D'après le corollaire 3.5, l'image $\operatorname{Im}\left(\rho_{\pi_{1}}\right)$ est un sous-groupe normal de $\pi_{1}(X)$ et elle est contenue dans le centre de $\pi_{1}\left(X_{\bar{k}}\right)$. Pour tout $k$-groupe fini $F_{1}$, d'après (1.5), tout $F_{1}$-torseur $Y_{1} \rightarrow X$ induit un homomorphisme $\theta_{1}: \pi_{1}\left(X_{\bar{k}}\right) \rightarrow F_{1}(\bar{k})$ à conjugaison près et, d'après la proposition 3.3 et le corollaire 3.5, $Y_{1}$ est $G$-compatible si et seulement si $\theta_{1} \circ \rho_{\pi_{1}}=0$.

D'après (1.5), soit $\alpha \in H^{1}\left(\pi_{1}(X), F(\bar{k})\right)$ un 1-cocycle qui correspond à $[Y] \in H^{1}(X, F)$. Il existe un sous-groupe ouvert distingué $\Delta \subset \pi_{1}(X)$ tel que $\left.\alpha\right|_{\Delta}=0$. Soient $\Delta_{\bar{k}}:=\Delta \cap \pi_{1}\left(X_{\bar{k}}\right)$ et $\alpha_{\bar{k}}:=\left.\alpha\right|_{\pi_{1}\left(X_{\bar{k}}\right)}$. Alors $\alpha_{\bar{k}}$ est un homomorphisme $\pi_{1}\left(X_{\bar{k}}\right) \rightarrow F(\bar{k})$.

Lemme 6.4. Pour trouver $Y^{\prime}$ dans la proposition 6.3, on peut supposer que $\Delta_{\bar{k}} \cdot \operatorname{Im}\left(\rho_{\pi_{1}}\right)=$ $\pi_{1}\left(X_{\bar{k}}\right)$ et donc $\operatorname{Im}\left(\alpha_{\bar{k}}\right)=\operatorname{Im}\left(\alpha_{\bar{k}} \circ \rho_{\pi_{1}}\right)$.

Démonstration. Le sous-groupe $\operatorname{Im}\left(\rho_{\pi_{1}}\right) \cdot \Delta$ est ouvert normal dans $\pi_{1}(X)$. Soit $Y_{2} \rightarrow X$ le revêtement galoisien correspondant. Par construction, $Y_{2} \rightarrow X$ est un torseur $G$-compatible sous un $k$-groupe constant $F_{2}=\pi_{1}(X) /\left(\operatorname{Im}\left(\rho_{\pi_{1}}\right) \cdot \Delta\right)$. Par hypothèse, il existe un $\sigma \in H^{1}\left(k, F_{2}\right)$ tel que $Y_{2, \sigma}\left(\mathbf{A}_{k}\right)^{\operatorname{Br}_{G}\left(Y_{2, \sigma}\right)} \neq \emptyset$. D'après le lemme 5.2 et (5.5), il existe une composante connexe $Y_{3} \subset Y_{2}$ telle que $Y_{3}$ est géométriquement intègre. Ainsi $Y_{3} \rightarrow X$ est un torseur sous un sousgroupe fermé $F_{3} \subset F_{2, \sigma}$ et on a

$$
\operatorname{Im}\left(\pi_{1}\left(Y_{3, \bar{k}}\right) \hookrightarrow \pi_{1}\left(X_{\bar{k}}\right)\right)=\pi_{1}\left(X_{\bar{k}}\right) \cap \operatorname{Im}\left(\pi_{1}\left(Y_{2}\right) \rightarrow \pi_{1}(X)\right)=\operatorname{Im}\left(\rho_{\pi_{1}}\right) \cdot \Delta_{\bar{k}}
$$

Alors $Y_{3} \rightarrow X$ est $G$-compatible. Par le lemme $6.2(3)$, après avoir remplacé $Y_{3}$ par son tordu, on peut supposer que $Y_{3}\left(\mathbf{A}_{k}\right)^{G \text {-ét, } \mathrm{Br}_{G}} \neq \emptyset$.

S'il existe un $\sigma \in H^{1}(k, F)$ et une composante connexe $Y_{3}^{\prime}$ du $F_{\sigma}$-torseur $Y_{\sigma} \times_{X} Y_{3} \rightarrow$ $Y_{3}$ tels que $Y_{3}^{\prime}$ soit géométriquement intègre, alors l'image de $Y_{3}^{\prime}$ par le morphisme fini étale $Y_{\sigma} \times_{X} Y_{3} \rightarrow Y_{\sigma}$ est une composante connexe $Y^{\prime}$ de $Y_{\sigma}$ telle que $Y^{\prime}$ soit géométriquement intègre. Donc on peut remplacer $X$ par $Y_{3}$ et, après avoir remplacé $X$ par $Y_{3}$, on peut supposer que $\Delta_{\bar{k}} \cdot \operatorname{Im}\left(\rho_{\pi_{1}}\right)=\pi_{1}\left(X_{\bar{k}}\right)$. Puisque $\alpha_{\bar{k}}\left(\Delta_{\bar{k}}\right)=0$, on a $\operatorname{Im}\left(\alpha_{\bar{k}}\right)=\operatorname{Im}\left(\alpha_{\bar{k}} \circ \rho_{\pi_{1}}\right)$.

Dans ce cas, puisque $\pi_{1}\left(G_{\bar{k}}\right)$ est commutatif, $\operatorname{Im}\left(\alpha_{\bar{k}}\right)$ est commutatif. D'après le corollaire 3.5, $\alpha_{\bar{k}}$ induit un homomorphisme $\pi_{1}\left(X_{\bar{k}}\right)^{a b} \rightarrow F(\bar{k})$ de noyau $\Gamma_{k}$-invariant, car $\alpha$ est défini sur $k$. D'après le corollaire 2.4, il existe un $k$-groupe fini commutatif $S$ et un $S$-torseur $\mathcal{T} \rightarrow X$ tels que $\mathcal{T}$ soit géométriquement intègre, $S(\bar{k})=\operatorname{Im}\left(\alpha_{\bar{k}}\right)$ et que, dans $H^{1}\left(X_{\bar{k}}, S\right) \cong$ $\operatorname{Hom}_{\text {cont }}\left(\pi_{1}\left(X_{\bar{k}}\right), \operatorname{Im}\left(\alpha_{\bar{k}}\right)\right)$, on ait $\left[\mathcal{T}_{\bar{k}}\right]=\alpha_{\bar{k}}$.

Soit $\left(H_{Y} \stackrel{\psi_{Y}}{\longrightarrow} G\right)$ le groupe minimal compatible au $F$-torseur $Y$. D'après la remarque 3.10, $\left(H_{Y} \stackrel{\psi_{Y}}{\longrightarrow} G\right)$ est aussi le groupe minimal compatible au $S$-torseur $\mathcal{T}$. D'après le corollaire 3.11, $\operatorname{Ker}\left(\psi_{Y}\right) \cong S$. Donc $Y_{4}:=\mathcal{T} \times_{X} Y$ est une $H_{Y}$-variété et $Y_{4} \rightarrow X$ est un $(S \times F)$-torseur $H_{Y}$-compatible. Donc $Y_{5}:=Y_{4} / \operatorname{Ker}\left(\psi_{Y}\right) \rightarrow X$ est un $F$-torseur $G$-compatible et on a un $F$ morphisme fini étale $\phi_{5}: Y_{5} \rightarrow Y / \operatorname{Ker}\left(\psi_{Y}\right)$. Par hypothèse, il existe un $\sigma \in H^{1}(k, F)$ tel que $Y_{5, \sigma}\left(\mathbf{A}_{k}\right)^{\operatorname{Br}_{G}\left(Y_{5, \sigma}\right)} \neq \emptyset$. D'après le lemme 5.2, il existe une composante connexe $Y_{5}^{\prime}$ de $Y_{5, \sigma}$ telle que $Y_{5}^{\prime}$ soit géométriquement intègre. Ainsi $\phi_{5}\left(Y_{5}^{\prime}\right)$ est une composante connexe de $\left(Y / \operatorname{Ker}\left(\psi_{Y}\right)\right)_{\sigma}$, qui est géométriquement intègre. Puisque $H_{Y}$ est connexe, les composantes connexes géométriques de $\left(Y / \operatorname{Ker}\left(\psi_{Y}\right)\right)_{\sigma}$ et de $Y_{\sigma}$ sont les mêmes, d'où le résultat.

Lemme 6.5. On a $X\left(\mathbf{A}_{k}\right)^{\text {ét, }^{-B_{G}}}=X\left(\mathbf{A}_{k}\right)^{G-e ́ t, B r} r_{G}$. 
Démonstration. Il suffit de montrer que, pour tout $x \in X\left(\mathbf{A}_{k}\right)^{G \text {-ét,Br}}{ }_{G}$, tout $k$-groupe fini $F$ et tout $F$-torseur $Y \stackrel{f}{\rightarrow} X$, il existe un $\sigma \in H^{1}(k, F)$, un $y \in Y_{\sigma}\left(\mathbf{A}_{k}\right)^{\operatorname{Br}_{H_{Y}}\left(Y_{\sigma}\right)}$ tels que $f_{\sigma}(y)=x$, où $\left(H_{Y} \stackrel{\psi_{Y}}{\longrightarrow} G\right)$ est le groupe minimal compatible au $F$-torseur $Y$.

On fixe de tels $x, F, Y, f$.

D'après la proposition 6.3, on peut supposer que $Y$ est géométriquement intègre.

D'après le corollaire 3.11, il existe un plongement $\phi: \operatorname{Ker}\left(\psi_{Y}\right) \rightarrow F$ d'image centrale compatible avec les actions de $\operatorname{Ker}\left(\psi_{Y}\right)$ et de $F$ sur $Y$. Ceci induit une suite exacte de $k$-groupes finis

$$
1 \rightarrow \operatorname{Ker}\left(\psi_{Y}\right) \stackrel{\phi}{\rightarrow} F \stackrel{\phi_{1}}{\rightarrow} F_{1} \rightarrow 1
$$

qui définit $F_{1}$. Alors $Y_{1}:=Y / \operatorname{Ker}\left(\psi_{Y}\right) \stackrel{f_{1}}{\longrightarrow} X$ est un $F_{1}$-torseur $G$-compatible sur $X$. De plus, $Y_{1}$ est lisse et géométriquement intègre.

Par hypothèse, il existe un $\sigma_{1} \in H^{1}\left(k, F_{1}\right)$ et un $y_{1} \in Y_{1, \sigma_{1}}\left(\mathbf{A}_{k}\right)^{\operatorname{Br}_{G}\left(Y_{1, \sigma_{1}}\right)}$ tels que $f_{1, \sigma_{1}}\left(y_{1}\right)=x$. D'après le corollaire 5.8, il existe un $\sigma_{0} \in H^{1}(k, F)$ tel que $\phi_{1, *}\left(\sigma_{0}\right)=\sigma_{1}$. Comme l'image de $\phi$ est centrale dans $F$, on a $\operatorname{Ker}\left(\psi_{Y}\right)_{\sigma_{0}}=\operatorname{Ker}\left(\psi_{Y}\right)$.

L'argument ci-dessus donne un $\operatorname{Ker}\left(\psi_{Y}\right)$-torseur $Y_{\sigma_{0}} \rightarrow Y_{1, \sigma_{1}}$ compatible avec l'action de $H_{Y}$. D'après la proposition 5.1, il existe un $\sigma_{2} \in H^{1}\left(k, \operatorname{Ker}\left(\psi_{Y}\right)\right)$ et un $y \in Y_{\sigma}\left(\mathbf{A}_{k}\right)^{\operatorname{Br}_{H_{Y}}\left(Y_{\sigma}\right)}$ avec $\sigma:=\sigma_{0}+\sigma_{2} \in H^{1}(k, F)$ tels que $f_{\sigma}(y)=x$.

Lemme 6.6. On a $X\left(\mathbf{A}_{k}\right)^{\text {ét,Br }}=X\left(\mathbf{A}_{k}\right)^{\text {ét, }, \mathrm{Br}}{ }_{G}$.

Démonstration. On peut supposer que $X\left(\mathbf{A}_{k}\right)^{\text {ét, } \mathrm{Br}_{G}} \neq \emptyset$. Il suffit de montrer que, pour tout $k$-groupe fini $F$ et tout $F$-torseur $f: Y \rightarrow X$, on a

$$
X\left(\mathbf{A}_{k}\right)^{\text {ét, } \operatorname{Br}_{G}} \subset \cup_{\sigma \in H^{1}(k, F)} f_{\sigma}\left(Y_{\sigma}\left(\mathbf{A}_{k}\right)^{\operatorname{Br}\left(Y_{\sigma}\right)}\right) .
$$

D'après la proposition 6.3, on peut supposer que $Y$ est géométriquement intègre. L'énoncé découle de la proposition 6.1 et du lemme 6.2 (1).

Démonstration du théorème 1.4. D'après le lemme 5.2 et (5.5), on peut supposer que $X$ est géométriquement intègre. On obtient le théorème par combinaison du lemme 6.6 et du lemme 6.5 .

Remarque 6.7. Rappelons les catégories AB et $\mathbf{G X}$ dans $\S 4$. On fixe un objet $(G, X) \in \mathbf{G X}$. Soit $\mathbf{G X}_{X}$ l'ensemble des objets $(H, Y) \in \mathbf{G X}$ tels qu'il existe un morphisme $(\psi, f)$ : $(H, Y) \rightarrow(G, X)$ dans $\mathbf{G X}$ avec $\psi, f$ finis.

Dans toute cette section (§6), pour établir le théorème 1.4 de $(G, X)$, l'hypothèse que $G$ est linéaire et la notion de sous-groupe de Brauer invariant sont utilisés seulement pour appliquer la proposition 3.12, la proposition 5.1, le corollaire 4.2, le lemme 5.2 et le corollaire 5.8 à l'élément dans $\mathbf{G X}_{X}$. Donc, cette section a essentiellement montré :

pour tout foncteur contravariant $B(-,-): \mathbf{G X} \rightarrow \mathbf{A B}$ qui associe au couple $(H, Y)$ un sousgroupe $B(H, Y) \subset \operatorname{Br}(Y)$, si l'on peut établir la proposition 3.12, la proposition 5.1, le corollaire 4.2, le lemme 5.2 et le corollaire 5.8 pour tout élément dans $\mathbf{G X}_{X}$ (en remplaçant tout groupe

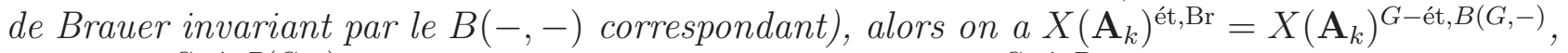
où $X\left(\mathbf{A}_{k}\right)^{G-e ́ t, B(G,-)}$ est défini de la même façon que $X\left(\mathbf{A}_{k}\right)^{G-e ́ t, B r}{ }_{G}$. 


\section{DÉmonstration Des ThÉORÈmes 1.1 ET 1.2}

Dans toute cette section, $k$ est un corps de nombres. Sauf mention explicite du contraire, une variété est une $k$-variété.

Démonstration du théorème 1.1. D'après le lemme 5.2 et (5.5), on peut supposer que $Z$ est géométriquement intègre. Si $G$ est connexe, l'énoncé découle du théorème 1.4 et de la proposition 5.5. Si $G$ est fini, d'après la proposition 6.3, on peut supposer que $X$ est géométriquement intègre, et le résultat découle du lemme 6.2 (2).

Pour établir le cas général, on reprend certains arguments de [D09a] et [CDX]. Il existe une suite exacte

$$
1 \rightarrow N \rightarrow G \stackrel{\psi}{\rightarrow} F \rightarrow 1
$$

de $k$-groupes linéaires avec $N$ un $k$-groupe linéaire connexe et $F$ un $k$-groupe fini. Alors

$$
h: U:=X / N \rightarrow Z
$$

est un $F$-torseur. Notons $q: X \rightarrow U$. Pour un $z \in Z\left(\mathbf{A}_{k}\right)^{\text {ét,Br }}$, il existe un $\sigma \in H^{1}(k, F)$ et un $u \in U_{\sigma}\left(\mathbf{A}_{k}\right)^{\text {ét,Br }}$ tels que $h_{\sigma}(u)=z$. D'après la proposition $5.7(1)$, il existe un $\alpha_{0} \in H^{1}(k, G)$ tel que $\psi_{*}\left(\alpha_{0}\right)=\sigma$. Ceci induit une suite exacte

$$
1 \rightarrow N^{\prime} \stackrel{\phi}{\rightarrow} G_{\alpha_{0}} \stackrel{\psi_{\alpha_{0}}}{\longrightarrow} F_{\sigma} \rightarrow 1
$$

de $k$-groupes linéaires. Alors $N_{\bar{k}}^{\prime} \cong N_{\bar{k}}$ et $N^{\prime}$ est un $k$-groupe linéaire connexe. Ainsi $q_{\alpha_{0}}$ : $X_{\alpha_{0}} \rightarrow U_{\sigma}$ est un $N^{\prime}$-torseur. Donc il existe un $\beta \in H^{1}\left(k, N^{\prime}\right)$ et un $x \in\left(X_{\alpha_{0}}\right)_{\beta}\left(\mathbf{A}_{k}\right)^{\text {ét,Br }}$ tels que $\left(q_{\alpha_{0}}\right)_{\beta}(x)=u$. Soit $\alpha:=\alpha_{0}+\phi_{*}(\beta)$. Alors $\left(X_{\alpha_{0}}\right)_{\beta}=X_{\alpha}$ et $p_{\alpha}=h_{\sigma} \circ\left(q_{\alpha_{0}}\right)_{\beta}$. Donc $x \in X_{\alpha}\left(\mathbf{A}_{k}\right)^{\text {ét,Br }}$ et $p_{\alpha}(x)=z$, d'où le résultat.

Démonstration du théorème 1.2. Ceci découle du théorème 1.1 et de [CDX, Thm. 1.5].

Démonstration $d u$ corollaire 1.5. Pour tout $k$-groupe fini $F$ et tout $F$-torseur $G$-compatible $f: Y \rightarrow X$, d'après le corollaire $3.5(4)$, il existe un $F$-torseur $M$ sur $k$ tel que $Y \cong M \times_{k} X$ comme $F$-torseurs. Alors il existe un $\sigma_{0} \in H^{1}(k, F)$ tel que $Y_{\sigma_{0}} \cong F \times X$. Donc

$$
X\left(\mathbf{A}_{k}\right)^{\operatorname{Br}_{G}(X)} \subset f_{\sigma_{0}}\left(Y_{\sigma_{0}}\left(\mathbf{A}_{k}\right)^{\operatorname{Br}_{G}\left(Y_{\sigma_{0}}\right)}\right) \subset \cup_{\sigma \in H^{1}(k, F)} f_{\sigma}\left(Y_{\sigma}\left(\mathbf{A}_{k}\right)^{\operatorname{Br}_{G}\left(Y_{\sigma}\right)}\right) .
$$

Ainsi $X\left(\mathbf{A}_{k}\right)^{G \text {-ét, } \operatorname{Br}_{G}}=X\left(\mathbf{A}_{k}\right)^{\operatorname{Br}_{G}(X)}$ et le résultat découle du théorème 1.4.

Remerciements. Je remercie très chaleureusement Jean-Louis Colliot-Thélène et Cyril Demarche pour leurs commentaires.

\section{RÉFÉRENCES}

[Ba] F. Balestrieri : Iterating the algebraic etale-Brauer set, Journal of Number Theory 182 (2018), 284-295.

[BD] M. Borovoi et C. Demarche : Manin obstruction to strong approximation for homogeneous spaces, Comment. Math. Hev. 88 (2013), 1-54.

[BS] M. Brion et T. Szamuely : Prime-to-p étale covers of algebraic groups and homogeneous spaces, Bull. Lond. Math. Soc. 45 (2013), no. 3, 602-612.

[Cod] B. Conrad : Weil and Grothendieck approaches to adelic points, Enseign. Math. 58 (2012), 61-97.

[CDX] Y. Cao, C. Demarche, F. Xu : Comparing descent obstruction and Brauer-Manin obstruction for open varieties, Trans. Amer. Math. Soc. 371 (2019), no. 12, 8625-8650. 
[C18] Y. Cao : Approximation forte pour les variétés avec une action d'un groupe linéaire, Compositio math. 154 (2018), 773-819.

[CLX] Y. Cao, Y. Liang, F. Xu : Arithmetic purity of strong approximation, Journal de Mathématiques Pures et Appliquées 132 (2019), 334-368.

[CT08] J.-L. Colliot-Thélène : Résolutions flasques des groupes linéaires connexes, J. reine angew. Math. 618 (2008), 77-133.

[CTSa] J.-L. Colliot-Thélène et J.-J. Sansuc : Principal homogeneous spaces under flasque tori, applications, Journal of Algebra 106 (1987) 148-205.

[CTSb] J.-L. Colliot-Thélène et J.-J. Sansuc : La descente sur les variétés rationnelles, II, Duke Math. J. 54 (1987) $375-492$.

[D09a] C. Demarche : Obstruction de descente et obstruction de Brauer-Manin étale, Algebra \& Number Theory 3 (2009) 237-254.

[D09b] C. Demarche : Méthodes cohomologiques pour l'étude des points rationnels sur les espaces homogènes, Thèse de doctorat, Université Paris-Sud (2009).

[Fu] L. Fu : Étale cohomology theory, Nankai Tracts in Mathematics, Vol. 13, World Scientific, 2011.

[HS02] D. Harari, A. N. Skorobogatov : Non-abelian cohomology and rational points, Compositio Math. 130 (2002) 241-273.

[HS13] D. Harari, A. N. Skorobogatov : Descent theory for open varieties, Torsors, étale homotopy and applications to rational points. LMS Lecture Note Series 405, Cambridge University Press (2013), $250-279$.

[KS] M. Kashiwara et P. Schapira, Categories and sheaves, Grundlehren der Mathematischen Wissenschaften, vol. 332, Springer-Verlag, Berlin, 2006.

[LX] Q. Liu et F. Xu Very strong approximation for certain algebraic varieties, Math. Ann. 363 (2015) $701-731$.

[Ma] Y. I. Manin : Le groupe de Brauer-Grothendieck en géométrie diophantienne, Actes du Congrès International des Mathématiciens (Nice, 1970), Tome 1, pp. 401-411. Gauthier-Villars, Paris, 1971.

[Mi] J. S. Milne : Étale Cohomology, Princeton Math. Ser 33, Princeton University Press, Princeton 1980.

[Miy] M. Miyanishi : On the algebraic fundamental group of an algebraic group, J. Math. Kyoto Univ. 12-2 (1972), 351-367.

[Po10] B. Poonen : Insufficiency of the Brauer-Manin obstruction applied to étale covers, Ann. of Math. 171 (2010) 2157-2169.

[Po] B. Poonen : Rational points on varieties, Graduate Studies in Mathematics, 186, American Mathematical Society, Providence, RI, 2017.

[S] J.-J. Sansuc: Groupe de Brauer et arithmétique des groupes algébriques linéaires sur un corps de nombres, J. reine angew Math. 327 (1981), 12-80.

[Se65] J.-P. Serre : Cohomologie Galoisienne, Lecture Notes in Mathematics, vol 5, Springer, Berlin, 1965.

[SGA1] A. Grothendieck et al. : Revêtements étales et groupe fondamental, (SGA 1), Lecture notes in mathematics 224, Berlin; New York, Springer-Verlag 1971.

[SGA4] A. Grothendieck, M. Artin, L. Verdier et al. : Théorie des topos et cohomologie étale des schémas, (SGA 4), Lecture Notes in Math. 269, 270, 305, Springer-Verlag (1972-1973).

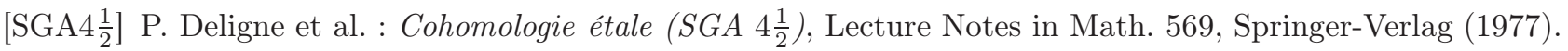

[Sk99] A. N. Skorobogatov : Beyond the Manin obstruction, Invent. Math. 135 (1999), no. 2, 399-424.

[Sk01] A. N. Skorobogatov: Torsors and Rational Points, Cambridge Tracts in Mathematics, vol. 144, Cambridge University Press, 2001.

[Sk09] A. N. Skorobogatov : Descent obstruction is equivalent to étale Brauer-Manin obstruction, Math. Ann. 344 (2009) 501-510.

[St] M. Stoll : Finite descent obstructions and rational points on curves, Algebra Number Theory 1 (2007) $349-391$. 
[SZ] A. N. Skorobogatov, Y. G. Zarhin : The Brauer group and the Brauer-Manin set of products of varieties, J. Eur. Math. Soc. 16 (2014) 749-769.

[Sz] T. Szamuely : Galois Groups and Fundamental Groups, Cambridge Studies in Advanced Mathematics 117, Cambridge University Press, Cambridge, 2009.

YANG CAO

LEIBNIZ UNIVERSITÄT HANNOVER

Welfengarten 1, 30167 Hannover, Allemagne

E-mail address: yang.cao@math.uni-hannover.de; yangcao1988@gmail.com 